\title{
Molecular characterization and transcriptional profiling suggested structural and functional divergence in ascorbate peroxidase (APX) and APX- related (APX-R) genes in Triticum aestivum $L$.
}

Shivi Tyagi

Panjab University

Shumayla .

Panjab University

Praveen Chandra Verma

National Botanical Research Institute CSIR

Kashmir Singh

Panjab University

Santosh Kumar Upadhyay ( $\nabla$ skupadhyay@pu.ac.in )

Panjab University https://orcid.org/0000-0001-6991-0502

\section{Research article}

Keywords: Ascorbate peroxidases; Co-expression; Enzyme activity; Paralogous gene; Transcriptional profiling; Triticum aestivum

Posted Date: November 4th, 2019

DOI: https://doi.org/10.21203/rs.2.16760/v1

License: (c) (1) This work is licensed under a Creative Commons Attribution 4.0 International License. Read Full License 


\section{Abstract}

Background: Ascorbate peroxidases (APXs) are heme-dependent $\mathrm{H} 2 \mathrm{O} 2$ scavenging enzymes providing the antioxidant defense and regulating myriad biological processes. APX-related (APX-R) is an additional form of APX protein which lacks peroxidase activity but form dimer with the chloroplastic APX (chIAPX). Despite of tremendous their functions, a systematic and comparative study of these genes is limited in Triticum aestivum L. Results: Herein, a total of 21 TaAPX and six TaAPX-R genes were identified from the A, B and D sub genomes of Triticum aestivum, which form nine homeologous groups. These identified genes consisted of three pairs of paralogous genes which confronted pre-hybridization gene duplication under purifying selection. An unequal evolutionary rate after gene duplication suggested functional divergence between paralogous genes. The phylogenetic study classified TaAPXs into four distinct clades, where TaAPX-R proteins were clustered in clade I. The members of each clade showed similarity in gene and protein architecture, motifs distribution, sub-cellular localization that suggested their conserved nature. The tertiary structure analysis of three TaAPXs and one TaAPX-R revealed the presence of helices and coils, and residues involved in ligand binding. Transcriptional profiling of TaAPX and TaAPX-R genes suggested their specific role during development and abiotic and biotic stress response. Modulated transcript expression and APX enzyme activity during heat, drought and salt stress confirmed their role in these stress conditions. Coexpression analyses predicted interaction of these genes with the other genes involved in numerous biological processes. These genes were also found to be regulated by miRNAs associated with various developmental pathways and/or stress response. Further, they also exhibited interaction with the mediator compounds of Halliwell-Asada cycle. Conclusions: Overall, the present study reported numerous molecular and structural features of TaAPXs and TaAPX-Rs, and their role in plant development and stress response. It provides a platform for detailed functional characterization of TaAPX and TaAPX-R genes in future studies.

\section{Background}

Ascorbate peroxidases (APXs) are class I heme peroxidases that belong to the non-animal peroxidase superfamily. These are abundant in the plant kingdom and metabolize hydrogen peroxide $\left(\mathrm{H}_{2} \mathrm{O}_{2}\right)$ into water $\left(\mathrm{H}_{2} \mathrm{O}\right)$ and oxygen $\left(\mathrm{O}_{2}\right)$ within the plant cell [1]. Moreover, they are also reported from Trypanosoma cruzi and bovine eye $[2,3]$. These are key regulatory enzymes of Halliwell-Asada or ascorbate-glutathione pathway that equilibrates the reactive oxygen species (ROS) concentration within the cell [4]. This pathway occurs in numerous sub-cellular organelles but it is crucial in chloroplast and mitochondria due to continuous production of ROS during photosynthesis and respiration $[5,6]$.

In a plant cell, APXs may occur in soluble or membrane-bound forms, and localized in various sub-cellular compartments [7]. They may be cytosolic (CAPX), stromal (SAPX) and mitochondrial (miAPX) in soluble form, while peroxisomal/glyoxisomal (mAPX) and thylakoidal (tAPX) in membrane-bound form [8]. A new form of APX called APX-related (APX-R) protein is also known, which forms a dimer with the chloroplastic APX (chIAPX) but lacks peroxidase activity [9]. They are conserved in most of the plant species and share high structural and functional homology. APXs show high specificity toward ascorbate (AsA) which acts as an electron donor during $\mathrm{H}_{2} \mathrm{O}_{2}$ reduction. The concentration of AsA within the cell extremely affects the APXs activity $[8,10]$. Moreover, cAPX and miAPX isoforms display comparable affinity toward other electron 
donors such as guaiacol or pyrogallol in certain conditions [1,11], which suggests the occurrence of two kinetically compatible binding sites in these isoforms [11].

In higher plants, $A P X$ genes are known for regulating the normal plant developmental processes and abiotic and biotic stresses as well by controlling the signal flux of $\mathrm{H}_{2} \mathrm{O}_{2}$. Under normal conditions, reduction in photosynthesis and growth rate, and delayed flowering has been observed in a CAPX1 mutant of Arabidopsis thaliana in comparison of wild type [12]. However, AtAPX3 mutant has no effect on growth and development [13]. Further, a $A P X-R$ gene i.e. AtAPX6 provides protection to seeds in A. thaliana from oxidative stress during desiccation, at the time of maturation and germination [14]. The function of OSAPX1 in seed development has been reported in Oryza sativa where mutant lines showed a reduction in panicle size and weight and produced $~ 58 \%$ aborted seeds. Moreover, the pattern of growth and development before flowering and number of seeds per panicle were similar to the wild-type [15]. The OSAPX2 knockout mutant has both pleiotropic effects on growth and development, and reduced tolerance to abiotic stress [16]. Higher APX activity is observed in a tolerant genotype of sweet potato after 24 and $48 \mathrm{~h}$ of salinity stress [8]. Further, an elevated expression of $A t A P X 2$ is reported at high light intensity and temperature stress [14]. Similarly, peroxisomal APX of Populus, OSAPX1, OsAPX2, and OSAPX5-OSAPX7 of O. sativa, and cytosolic APX2 of Vigna unguiculata exhibited elevated expression during drought stress [8]. Over expression of $t A P X$ of Solanum lycopersicum provided enhanced tolerance to salt and osmotic stress in transgenic tobacco plants [8].

Despite their significant roles, a systematic characterization of $A P X$ genes (TaAPXs) has not been carried out in the staple crop Triticum aestivum (bread wheat). In the current study, we performed genome-wide analysis of TaAPX and TaAPX-R genes in T. aestivum. The identified genes and proteins were subjected to a systematic in-silico characterization to explore their physicochemical and structural properties. The phylogenetic and syntenic relationships, gene duplication events and divergence time of paralogous genes were estimated to understand the evolution of $A P X$ and $A P X-R$. The cis-regulatory elements, miRNA targets, and expression and co-expression patterns were studied to reveal the functions of these genes in growth and development and stress conditions. Further, the expression of randomly selected one gene from each phylogenetic clade was confirmed by qRT PCR. The APX enzyme assay was also performed after heat, drought and salinity stress treatments to validate their role in these stresses.

\section{Results}

\section{Identification and chromosomal localization of TaAPX and TaAPX-R genes}

A total of $21 T a A P X$ and six TaAPX-R genes were identified from the A, B and D sub-genomes of $T$. aestivum, which were clustered into nine homeologous groups (HGs) (Additional file 1). These HGs included seven TaAPX (TaAPX-1 to TaAPX-3, TaAPX-5 to TaAPX-7, and TaAPX-9) and two TaAPX-R (TaAPX-4 and TaAPX-8) group genes. A maximum of four genes (three TaAPXs and one TaAPX-R) were localized on chromosome 2, which was followed by chromosome 7 with two genes on each $A, B$ and $D$ sub-genome. The remaining chromosomes consisted of one gene each except chromosome 1 and 3 (Fig. 1a, Additional file 2). 
The study of paralogous genes suggested the occurrence of three segmental gene duplication events (TaAPX2-A/ TaAPX5-A, TaAPX2-B/ TaAPX5-B, and TaAPX2-D/TaAPX5-D) with more than $5 \mathrm{Mb}$ distance between paralogous gene pairs (Fig. 1a, Table 1). The Ka/Ks ratio of each duplicated gene pair was $<0.1$, which indicated purifying selection. Analysis of divergence time indicated the occurrence gene duplication around 79 to 93 mya (million years ago) (Table 1). The evolutionary rate per year between the duplicated gene pairs was compared using Tajima's relative rate test. The results showed a significant value (30-44) of $\chi 2$ for all duplicated gene pairs (Table 2). Further, a diverse expression pattern was observed between the duplicated genes (Fig. 1b).

\section{Phylogeny and synteny analysis}

A total of eight $A P X$ genes in each $A$. thaliana, Brachypodium distachyon, and Hordeum vulgare, and ten in each $O$. sativa, and Zea mays were identified through extensive data mining and used for the analysis of syntenic relationship with $T a A P X$ and $T a A P X-R$ genes (Fig. 2a-e). The results indicated variable distribution of these genes on various chromosomes in each plant species, and a close evolutionary relationship among the orthologs of different plant species. The APX protein sequences of these orthologs were used for the phylogenetic analysis (Fig. 3). Tree topology indicated the grouping of APXs into four distinct clades, which were further divided into sub-clades (Fig. 3). Based on the homology with the earlier reported APX proteins of $A$. thaliana and $O$. sativa, it was presumed that the clade I APXs were mostly chloroplastic, and specific to a special group of APX-related (APX-R) proteins $[9,17]$. However, APXs of clade II, III and IV were predicted as cytoplasmic, peroxisomal and chloroplastic/mitochondrial, respectively.

\section{Intron/exon distribution and splice variants}

Gene structure of all TaAPXs and TaAPX-Rs and their splice variants were investigated for exon/intron organization and intron phases. The number of exons ranged from 7 to 12 , however, $46 \%$ of genes were having 11 exons (Fig. 4a). Among all the genes, $~ 60 \%$ introns were in 0 intron phase, followed by $23 \%$ and $17 \%$ in phase 1 and 2, respectively. TaAPX-3, TaAPX-6 and TaAPX-7 group genes predominantly consisted of 0 phase introns, while intron phase 1 and 2 were leading in case of $T a A P X-8$ group genes. TaAPXs also shared the conserved pattern in the number and length of various exons across the tightly clustered genes in phylogeny. For instance, the TaAPX1, TaAPX2, TaAPX5 and TaAPX9 group genes, and TaAPX3, TaAPX6 and $T a A P X 7$ group genes shared a similar number and length of exons. However, TaAPX4 and TaAPX 8 group genes of $T a A P X-R$ exhibited different genomic structure as compared to the other group genes. The length of exon 2, 4, 5, 6 and 7 was conserved with 125, 49, 83, 80 and 103 bp, respectively. However, the 3rd exon (116 bp) of $T a A P X-1$ was assumed to be split between $3 \mathrm{rd}(50 \mathrm{bp})$ and 4 th (66 bp) exons of TaAPX-9 group genes, or vice versa. Similarly, 2nd (125 bp) and 3rd (50 bp) exons of TaAPX9 were probably merged to form 2nd exon (175 bp) of TaAPX-2 and TaAPX-5 group genes or vice versa. Moreover, the length of other exons was conserved in these genes. On the basis of variation in the number of exons or exon length, a total of 13 and three splice variants were identified, which were encoded by nine TaAPX and three TaAPX-R genes, respectively (Fig. 4a, Additional file 1). The TSA BLAST analysis suggested the existence of most of the splice variants at transcript stage (Additional file 3). Nine splice variants differed in exon length, while seven in exon number. The difference in length of the 2nd exon was observed in most TaAPX- 6 group genes and their splice variants. TaAPX3-A. 1 and TaAPX3-A.2 showed the difference of three nucleotides in the length of 
the 9th exon. However, a few have differences in higher nucleotide numbers such as 1 st exon of TaAPX7-B.1 and TaAPX7-B.2 differed in 24 nucleotides. In case of variable number of exons, the events of exon skipping such as in TaAPX9-D.2 or intron retention such as in TaAPX7-B.3 were observed. However, the other variants differed in the number of exons such as TaAPX5-A.1, TaAPX8-A.1, TaAPX8-D.2 etc. (Fig. 4a).

\section{Physicochemical and structural properties}

The length of TaAPXs and TaAPX-Rs ranged from 227 to 469 amino acid (AA) residues (Table 3, Additional file 2). The average molecular weight (MW) of clade I (TaAPX4 and TaAPX8), clade II (TaAPX2 and TaAPX5), clade III (TaAPX1 and TaAPX9) group proteins were 35, 29 and $31 \mathrm{kDa}$, respectively. In clade IV, the average MW of TaAPX3 and TaAPX6 group proteins was $35 \mathrm{kDa}$, while TaAPX7 group proteins was $47 \mathrm{kDa}$. Latter consisted of an additional transmembrane helix (TM). The presence of TM was also predicted in clade III proteins or mAPXs except TaAPX1-B and TaAPX9-D.2. The pl ranged from 4.9 to 9.25, though the pl of maximum proteins was above 7 . The grand average hydropathy (GRAVY) value was ranged from -0.522 to -0.108 . The minutiae of protein sequences were analyzed for conserved motifs using the MEME server as well as multiple sequence alignments (Fig. $4 \mathrm{~b}$ and $5 \mathrm{a}-\mathrm{d}$ ). The TaAPX proteins showed a conserved pattern in various AA residues involved in catalysis and ligands binding such as Lys-30, Cys-32, Arg-172, Trp-179, His163 etc. However, in case of TaAPX-Rs most of these residues such as Cys-32, Arg-172, Trp-179 etc. were either absent or substituted with the other AA residues. Further, TaAPX-4 and TaAPX-8 group proteins were also varied from each other in the primary structure at the active-site domain (ASD), heme-binding domain (HBD) and cation-binding domain (CBD) (Fig. 5d). The MEME server identified a total of ten conserved motifs in the TaAPX proteins (Fig. 4b, Additional fig. 1). Motifs 1 to 9 were found conserved in all chlTaAPX proteins (TaAPX3, TaAPX4 and TaAPX7), while motif 10 was additionally present in TaAPX7 group proteins (Fig. 4b). In mAPX (TaAPX1 and TaAPX9) and cAPX (TaAPX2 and TaAPX5), all the motifs were conserved, except motifs 6 and 10. Motifs 2, 5, 7 and 8 were conserved in TaAPX-R (TaAPX4 and TaAPX8) proteins. Analyses of AA residues of motifs 2,3 and 6 showed that they represent ASD, HBD, and CBD, respectively. The BLAST search of the other motifs sequence revealed the presences of heme-dependent peroxidase domain. Further, two conserved signature motifs; chloroplastic domain 1 (K-N-I-[EKNS]-[ED]-W-P) near ASD, and chloroplastic domain 2 (E-T-K-Y-T-K-[DNTE]-G-P-G-[AE]-[PA]-G-G-Q-S) near HBD were also detected in chITaAPX proteins.

The secondary and tertiary structure analyses revealed the prominence of helices and loops in TaAPX proteins (Fig. 5e-5h). The percentage of helices was higher than loops in TaAPX1-A (mAPX) and TaAPX2-B (CAPX) in contrast to TaAPX3-A.1 (chIAPX) and TaAPX8-B.1 (APX-R). Primarily, helices A to $\mathrm{J}$ were present in all the structures, however, few small helices such as $A 1^{\prime}, B^{\prime}, D^{\prime}$ etc. were also identified adjacent to some helices. Further, TaAPX1-A, TaAPX2-B and TaAPX8-B.1 have common best PDB hit (2vcfX, cAPX of soybean), but TaAPX2-B showed high percentage structural homology with 2vcfX than others, indicating the subcellular localization based structural relatedness. Besides this, TaAPX3-A.1 showed high homology with PDB hit 1 iynA, a chIAPX from the tobacco plant. Various structural parameters and ligand binding residues have been mentioned in Table 4. Further, TaAPX3 and TaAPX7 group proteins and TaAPX-R (TaAPX4 and TaAPX8) proteins consisted of cTP while the TaAPX6 group proteins consisted of mTP (Additional file 2). TaAPX1 and TaAPX9 group proteins consisted of an alanine and valine rich region along with SRK domain (Fig. 5b) which suggested their peroxisomal localization $[18,19]$. 


\section{Promoter analysis}

The promoter region of all TaAPX and TaAPX-R genes were analysed for the presence of various cisregulatory elements except TaAPX4-B and TaAPX8-A due to unavailability of the promoter sequence. They have been broadly categorized into growth and development related, hormone, light, and stress-responsive cis-acting elements (Additional table 1). The growth and development responsive elements such as CCGTCCbox and CCGTCC-motif related to meristem-specific activation were predominant among these genes. The cis-acting elements such as circadian, AC-I, AC-II, CAT-box, $\mathrm{O}_{2}$-site etc., were also identified in the promoter region of various genes and known for regulating different plant developmental processes. However, none of the growth and development responsive elements has been identified in TaAPX5-A and TaAPX8-D. The hormone responsive elements such as CGTCA-motif and TGACG- motif involved in the MeJA responsiveness, and MYC involved in jasmonic and abscisic acid signalling were prominent in the majority of the promoter. Besides this, AuxRR-core and TGA-element (auxin-responsive elements), and GARE-motif, Pbox and TATC-box (gibberellin-responsive element) were also found in the promoter region of certain TaAPX genes. Various kind of light responsive cis-acting elements such as ATC-motif, AE-box, Box III, Box 4, G-box, GATA-motif, Gap-box, GATT-motif, TCCC-motif, TCT-motif etc. have also been found in TaAPXs promoters. Additionally, several stress-responsive cis-acting elements were identified including ABRE (abscisic acid responsive element), as-1 (involved in stress response related to auxin, salicylic acid, and methyl jasmonate), MYB (drought inducibility), MBS (MYB binding site; involved in drought-inducibility), STRE (heat, osmotic, starvation etc. stress responsive), W box (defence against pathogen), WRE3 (wound-response element) etc.

\section{Transcriptional profiling in tissue developmental stages and stress conditions}

The transcriptional profiling was performed to analyze the putative role of $T a A P X$ and $T a A P X-R$ genes during plant development and in the presence of various biotic and abiotic stress conditions. A significant correlation ( $\mathrm{R}^{2}$ 0.98) between the replicates suggested consistent expression value in replicates (Fig. 6a, b). During plant development, TaAPX1, TaAPX2, TaAPX3 and TaAPX5 group genes showed spatiotemporal expression in different developmental stages of root, stem, spike and grain (Fig. 6c), in which former three were highly expressed in spike, grain and root tissues, respectively. However, TaAPX7 and TaAPX9, and $T a A P X-R(T a A P X 4$ and $T a A P X 8)$ group genes were predominantly expressed in all the leaf developmental stages and stem_z65. Moreover, high expression of TaAPX1,TaAPX2 and few other genes were observed in spike and grain developmental stages.

During biotic stress, the expression of a total of 25 transcripts including splice variants was affected (Fig. 6d). TaAPX3-B, TaAPX3-D and TaAPX5-A.2 were consistently up-regulated after $B g t$ infestation, but less affected after Pst infestation. However, TaAPX6-A.1, TaAPX6-B.1, TaAPX6-B.3 and TaAPX8-A.2 were upregulated after the infestation of both the fungal pathogens. A few genes were found to be either early or late responsive. For instance, TaAPX3-A.2 and TaAPX7-B.2 were upregulated after $24 \mathrm{~h}$, while TaAPX6-B.1 and TaAPX8-A.2 were upregulated after $72 \mathrm{~h}$ of infestation of $B g t$ and $P s t$, separately. Further, a few genes were found to be pathogen-specific such as $T a A P X 5-D$ and $T a A P X 1-B$ were exclusively up- regulated after $B g t$ and Pst infestation, respectively. 
A total of 13 transcripts exhibited differential expression in the presence of salt stress, and most of them showed temporal differentiation (Fig. 6e). For instance, TaAPX6-B.3 and TaAPX6-D.2; TaAPX3-B; TaAPX5-B.2 and TaAPX6-D.3; TaAPX1-A, TaAPX5-A. 1 and TaAPX6-A. 1 were selectively upregulated at 6, 12, 24 and $48 \mathrm{~h}$ of salt treatment, respectively. TaAPX1-B and TaAPX8-D.2 were found upregulated at both 24 and $48 \mathrm{~h}$, and TaAPX8-A. 1 at 6 and $24 \mathrm{~h}$ of salt treatment. However, TaAPX6-D. 1 and TaAPX6-D. 2 were down-regulated at 24 and $48 \mathrm{~h}$ of salt treatment.

The transcriptional profiling of TaAPX genes was also performed after heat (HS), drought (DS) and their combination (HD) stress. Expression of a total of 29 transcripts was affected during these stresses (Fig. $6 \mathrm{f}$ ). The majority of $T a A P X$ and $T a A P X-R$ genes were downregulated after HS, DS and HD, while TaAPX1-A, TaAPX1-B, TaAPX1-D TaAPX7-B.3 and TaAPX8-D.2 were upregulated in one or more stress. TaAPX7-B.2 was upregulated in each treatment except DS_1h, while TaAPX3-D was downregulated in all treatments.

The validation of expression profile of one randomly selected gene from each phylogenetic clade such as TaAPX3-B, TaAPX5-B, TaAPX8-D and TaAPX9-A was performed by qRT PCR under similar abiotic stress conditions. The results were mostly found consistent with the expression pattern obtained from the RNA seq data (Additional Fig. 2, Additional table 2).

\section{Co-expression analysis}

The co-expression analyses of TaAPX and TaAPX-R genes were performed to explore their putative modusoperandi in tissue developmental stages and stress conditions, as well. A total of 51 genes were found coexpressed with TaAPX6-D.3 and 5 genes with TaAPX6-A. 1 in different tissue developmental stages (Fig. 7a). However, under biotic, salt, and heat, drought and their combination stress in total 74, 130 and 105 genes were co-expressed with 7, 16, $18 T a A P X$ and TaAPX-R genes, respectively (Fig. 7b-d). The co-expressed genes encode various development related and stress-responsive proteins such as wall-associated receptor kinase (WAK), E3 ubiquitin-protein ligase (E3-Ubl), F-box, chaperone protein DnaJ, mitogen- activated protein kinase kinase 3-like isoform X1 (MAPKK3), receptor-like cytoplasmic kinase 176 (RLCK176), dehydrin 7 (dhn7), auxin response factor 17 (ARF17), histone proteins, serine/threonine-protein kinase (STK), WRKY transcription factor 72 (WRKY72), GDSL esterase/lipase (GDSLE), blue copper protein (BCP) etc. (Additional file 4). The gene ontology (GO) mapping of these co-expressed genes suggested their role in the biosynthetic process, reproduction, cell differentiation, growth, response to stress, protein kinase activity, thiol-dependent ubiquitin-specific protease activity, protein hetero-dimerization activity, defence response to fungus etc. (Additional file 4).

\section{miRNAs and chemical interaction}

A total of 51 known miRNAs of $T$. aestivum showed interaction with $29 T a A P X$ and nine $T a A P X-R$ transcripts including splice variants, where 44 miRNAs function through cleavage while six miRNAs by translation inhibition (Fig. 7e, Additional file 5). Moreover, tae- miR319b functions by both mechanisms, it regulates the expression of TaAPX6-B and TaAPX6-D by cleavage, and TaAPX4-D and TaAPX4-A by translation inhibition. A few miRNAs including tae-miR2026a, tae-miR1211a, tae-miR444b etc. target single gene, while the majority of miRNAs interacted with more than one TaAPX genes. For instance, tae- miR319b and tae-miR396g 
targeted TaAPX4-A, TaAPX4-D, TaAPX6-B and TaAPX6-D, and TaAPX4-A, TaAPX4-B, TaAPX4-D, TaAPX5-A, TaAPX5-B and TaAPX5-D, respectively.

The chemical interaction analysis showed the association of TaAPX and TaAPX-R proteins with most of the mediator compounds in Halliwell-Asada or ascorbate-glutathione cycle such as $\mathrm{H}_{2} \mathrm{O}_{2}$, ascorbic acid, catalase, ascorbate radical, reduced glutathione, dehydroascorbic acid, superoxide anion, GSSG etc. [4]. Besides this, the association with other inorganic compounds like calcium ions, water, manganese, sodium, ammonia, oxygen was also observed (Fig. 7f, Additional file 6).

\section{Ascorbate peroxidase assay}

The effect of HS, DS, HD and salt stress on APX activity was analyzed in root and shoot tissues of $T$. aestivum. The APX activity in shoot tissue was significantly increased by $~ 2$ fold at $1 \mathrm{~h}$ of HS and DS treatments, while 1.5 fold in HD $1 \mathrm{~h}$ (Fig. 8a). However, the activity was 1.5 fold higher at $6 \mathrm{~h}$ of HS, DS and HD treatments. The activity in root tissue was significantly increased at DS $6 \mathrm{~h}$, whereas, it was not much affected in case of HS and HD (Fig. 8b).

In case of salt stress, the activity in shoot tissue was increased by 1.3 fold at $12 \mathrm{~h}$ and $24 \mathrm{~h}$ but remained unaffected at $6 \mathrm{~h}$. However, at $48 \mathrm{~h}$ the activity decreased by 1.2 fold (Fig. 8c). Similarly, the APX activity in root tissue was increased $\sim 1.3$ fold in 6,12 and 24 h of treatment while the activity declined by $\sim 1.2$ fold at $48 \mathrm{~h}$ of treatment (Fig. 8d).

\section{Discussion}

APX provides antioxidant defense response and regulates various biological pathways by maintaining $\mathrm{H}_{2} \mathrm{O}_{2}$ homeostasis within the plant cell. The present study focuses on the molecular characterization of TaAPX and $T a A P X-R$ genes and proteins in the genome of $T$. aestivum. It reinforces the knowledge about the structure, phylogenetic and syntenic relationships, and transcriptional profiling of $T a A P X s$ and $T a A P X-R$. The APX assay was also performed to examine the change in enzyme activity under various abiotic stress treatments.

The studies in different plants showed the occurrence of six to eight APX genes in most of the diploid plants such as in A. thaliana, Gossypium raimondii, O. sativa, etc. [19-21], while 26 GhAPX genes in tetraploid G. hirsutum [22]. In lower plants, the occurrence of four and five genes were reported in Chlamydomonas reinhardtii and Physcomitrella patens, respectively [21]. In the present study, 27 TaAPX genes including $T a A P X-R$ are identified in $T$. aestivum. The occurrence of about thrice the number of $A P X$ genes in the $T$. aestivum genome as compared to the diploid plant species might be attributed to their allohexaploid nature. The results suggested an analogy in the number of $A P X$ genes and the complexity of the plant genome. On the contrary, typically one $T a A P X-R$ gene has been reported in most of the plants and a few consisted of two genes [23]. This might be attributed to gene loss during the course of evolution.

In addition to the ploidy level, the expansion of gene families occurs due to the phenomenon of gene duplication, which is important for genetic diversity. Certain duplicated genes showed functional divergence 
while others remain conserved based on their evolutionary rate [22]. In the various plant species, one to three segmental DEs are reported in $A P X$ gene family [21] as observed in the case of $T$. aestivum. However, a few tandem, or both segmental and tandem DEs were also described in certain plants such as $B$. distachyon, $G$. raimondii, Z. mays etc. [21]. The TaAPX-R genes faced strong negative selection pressure; therefore, gene duplication among them is uncommon [23]. Similar to the TaAPXs, the paralogous $A P X$ genes in other plant species such as $G$. hirsutum were also under purifying selection pressure, which indicated conserved nature of this gene family [22]. The hybridization events among the progenitors of $A, B$ and $D$ sub genomes of $T$. aestivum were predicted from 0.5 to 6 mya [24], which suggested pre-hybridization DEs among TaAPX genes. The significant value of $\chi 2$ within duplicated genes indicated that the genes were evolved at an unequal rate. It leads to the rejection of the molecular evolutionary clock hypothesis which articulate a similar evolutionary rate for the duplicated gene [25]. The deviations in the expression profiles of duplicated $T a A P X$ gene pairs also supports the incidence of functional divergence or neo-functionalization among the paralogous genes. In the case of G. hirsutum, GhAPX10A/11A/12A duplicated genes were significantly evolved at an unequal rate suggesting the occurrence of functional divergence. However, GhAPX10D/11D/12D duplicated genes showed insignificant evolutionary rate [22]. The synteny and phylogenetic analysis indicated a close evolutionary relationship among the orthologous genes of various plant species. The phylogenetic clustering of APXs with respect to their sub-cellular localization has also been discussed in several plant species such as $O$. sativa and $G$. hirsutum $[19,22]$. APX-R proteins were separately clustered and are known to control the activity of other APXs especially chIAPXs through physical protein-protein interaction and regulate antioxidant responses [10].

The occurrence of comparable intron/exon organization and similar exon length in the APX genes of various plant species such as $A$. thaliana, $O$. Sativa, Pisum sativum, $T$. aestivum and other plants suggested conserved gene architecture in higher plants $[19,21,22]$. However, variations in the structure of $T a A P X-R$ genes suggested their divergent nature in comparison to the TaAPX genes. The analysis of splice variants indicated the events of exonization or pseudo-exonization during the course of evolution of this gene family. The variation in exon length in splice variants was probably introduced due to the availability of additional $5^{\prime}$ or 3 ' splice sites. The occurrence of splice variants may facilitate structural and functional divergence among TaAPX genes.

The physicochemical and structural properties of TaAPX and TaAPX-R proteins show consistency with other plant species such as $A$. thaliana, $B$. distachyon, G. max, $O$. sativa, Z. mays etc. [19, 21, 22]. Further, the low GRAVY value of TaAPXs suggested their hydrophilic nature and better interactions with water. APX also shares highly conserved motifs and domains such as ASD, HBD and CBD which are important for the peroxidase activity. These domains are substituted with new sets of conserved amino-acid residues in APX-R [9]. In addition, the two signature motifs specific for chIAPXs play a significant role in determining the substrate specificity, but they are not related to peroxidase activity [19]. The occurrence of motif 10 at Cterminus of chITaAPX7 and N-terminus of TaAPX8 group proteins suggested their possible interaction as shown in chIAPX and APX-R of $O$. sativa [9]. The localization of APXs into different subcellular compartments mainly depends on the presence of a type of sorting peptide such as cTP and MTP for chloroplast and mitochondria, respectively. However, the peroxisomal APX usually consist of alanine and valine rich hydrophobic trans-membrane region followed by a positively charged domain $[18,19]$. The 
peroxisomal localization of APX proteins usually depends upon the overall basic AAs composition rather than the presence of a specific sequence or a single positively charged AA residue [18].

The presence of development related and stress-responsive cis-acting elements in the promoter region of $T a A P X s$ and $T a A P X-R$ genes suggested their role in various biological processes and defense responses. The occurrence of related or similar cis-acting elements has been described in the promoter region of TaSODS, TaGPXs and GhAPX genes etc. [22, 26, 27]. These cis-acting elements suggest the co-activation of genes involved in ROS detoxification and other antioxidant mediated biological pathways. The specifically high expression of certain $T A A P X$ genes in particular tissues suggested their precise role in its growth and development such as involvement of TaAPX6 during grain maturity, and TaAPX1 and TaAPX2 in the flowering, reproduction and grain filling. The function of AtAPX1 in plant growth and flowering, and AtAPX6 in seed development has been described in A. thaliana [12, 14]. APXS are also known to regulate leaf development and senescence, root development, pre and post-reproduction processes, cell elongation etc. through signaling mechanism [28].

Besides this, the importance of $A P X s$ in defense response during biotic and abiotic stresses is well known in numerous plants $[8,29]$. In case of 0 . sativa, OSAPX8 is involved in providing tolerance against bacterial blight, while $O S A P X 1$ and $O S A P X 2$ have been reported in providing tolerance against pathogen attack, oxidative and salinity stress [29]. Further, the enhanced expression of $C A P X$ in response to wounding has been shown in sweet potato [30]. The pathogen-specific expression of TaAPX genes suggested distinct responses of these genes during biotic stress. The expression analyses of TaAPX and TaAPX-R genes during HS, DS, HD and salt stress suggested their role in abiotic stress tolerance. The function of $A P X$ genes in providing salinity tolerance has been earlier described in numerous plant species such as $A$. thaliana $O$. sativa, S. lycopersicum etc. [29]. Similarly, the upregulation of APX under heat, drought and salt stresses has been reported in Brassica campestris, H. vulgare and O. sativa [29]. The over expression of B. campestris and $H$. vulgare APX genes showed enhanced thermal tolerance in $A$. thaliana [31]. The activity of APX also gets affected under different stress conditions in T. aestivum, as reported in case of other plants. For instance, in rice and soybean the activity of APX was higher in salt tolerant genotypes compared to susceptible genotype $[32,33]$. While, in some cases such as rice the activity of APX was higher in sensitive genotype [34]. Under drought stress condition the higher APX activity was observed in soybean [35]. Similarly, the enhanced APX activity was observed in various plant species under different stress conditions like $A$. thaliana, Ipomoea batatas, Medicago sativa, S. lycopersicum, S. tuberosum, O. sativa, G. hirsutum etc. [29]. Similarly, we observed increased APX activity during most of the analyzed abiotic stress conditions in shoot and root tissues of T. aestivum.

The co-expression analysis suggested the interaction of $T a A P X$ and $T a A P X-R$ genes with the other genes involved in diverse functions. Further, a few co-expressed genes also showed interaction with the other antioxidant enzymes encoding genes such as TaGPX [27], indicated their role in signalling during ROS burst. The regulation of TaAPX through miRNAs also strengthened their involvement in various biological pathways. These miRNAs were reported to target various plant development and stress-responsive genes in earlier studies. For instance, tae-miR1211a targets superoxide dismutase [Cu-Zn] 4AP, serine/threonineprotein kinase WNK2, Fumarate hydratase 2, SNF1-related protein kinase regulatory subunit beta-1-like etc., 
tae-miR444b targets MADS- box transcription factor, tae-miR390a and tae-miR390b targets ARF (auxinmediated transcriptional activation/suppression), tae-miR396 targets growth-regulating factor 9 , taemiR319b targets transcription factor PCF etc. [36]. Further, the interactions of TaAPXs with various chemical compound suggested that they might functions as co-factors of either APX or interacting enzymes/proteins. However, the functional validation of each interaction needs to be performed in future studies.

\section{Conclusions}

In conclusions, structure analysis suggested conserved nature of TaAPX and TaAPX-R genes and proteins. Despite of structural similarities, the paralogous $\operatorname{Ta} A P X$ genes were evolved at an unequal evolutionary rate and exhibited functional divergence. Differential expression profiling in developmental stages and stress conditions revealed their diverse biological functions, which were also supported by their co-expression with the other development related and stress responsive genes. These genes were also found to be regulated by miRNAs and various other factors. The stress responsive behavior of these genes might be explored for the development of stress resistant transgenic crop plants in future studies. Further, the interaction of TaAPX and TaAPX-R genes with each other as well as with the other genes can also be established.

\section{Methods}

\section{Identification of TaAPX and TaAPX-R genes and their chromosomal positions}

The TaAPX and TaAPX-R genes were identified by BLASTp search using known APX sequences from $A$. thaliana and $O$. sativa as a query against $T$. aestivum gene and protein model sequences (IWGSC RefSeq assembly v1.0.) downloaded from Ensembl Plants (http://plants.ensembl.org/index.html). The identified sequences were used for Hidden Markov Model (HMM) search for the peroxidase domain (PF00141) using Pfam BLAST at e-value $10^{-10}$ [37]. The non-redundant set of genes was then confirmed through PeroxiScan search [38]. NCBI Conserved Domain Database search was used to reconfirm the legitimacy of APX domain in each TaAPX and TaAPX-R sequence [39]. The existence of identified genes at transcript level was confirmed using BLASTn search against transcriptome shotgun assembly (TSA) sequences of $T$. aestivum at NCBI server (https://www.ncbi.nlm.nih.gov/genbank/tsa/). The nomenclature of identified TaAPX and $T a A P X-R$ genes was carried out following the guidelines of international rules of genetic nomenclature for gene symbolization in wheat (https://wheat.pw.usda.gov/ggpages/wgc/98/Intro.htm). The plausible homeologous genes were identified through the standard procedures including bidirectional BLAST search $\left(10^{-10}\right.$ e-value and $\geq 90 \%$ identity) and chromosomal similarity, as reported earlier [40, 41]. However, the similarity of $\geq 80 \%$ among intra sub-genomic genes was used for the identification of paralogous genes. The localization of these genes on various chromosomes was retrieved from Ensembl Plants [42] and represented using Maplnspect (https://mapinspect.software.informer.com/).

\section{Evolutionary and syntenic relationships}

The orthologous genes were identified through bi-directional BLAST search (e value $10^{-10}$ ) in $A$. thaliana, $B$. distachyon, $H$. vulgare, $O$. sativa, and $Z$. mays. The non-redundant full-length protein sequences were aligned using MUSCLE [43] and a phylogenetic tree was constructed at 1000 bootstrap value by Neighbor-Joining 
(NJ) method using MEGA7 [44]. The syntenic relationship among orthologous was depicted using the Circos0.69-6 software [45].

\section{Calculation of $\mathrm{Ka} / \mathrm{Ks}$, divergence time and Tajima's relative rate test}

The nucleotide and peptide sequences of duplicated gene pairs were aligned using the ParaAT2.0 [46]. The alignment was used for the estimation of the number of non-synonymous substitutions per nonsynonymous site (Ka), synonymous substitutions per synonymous site $(\mathrm{Ks})$ and the ratio of $\mathrm{Ka} / \mathrm{Ks}$ using the KaKs Calculator [47]. The formula, $T=K s / 2 r$ was used for the calculation of divergence time between the duplicated genes, where $T$ and $r$ represent the divergence time and divergence rate, respectively. For monocotyledonous plants, the divergence rate was presumed to be $6.5 \times 10^{-9}$ [48]. Additionally, the Tajima's relative rate test was performed to analyze the relative evolutionary rate after gene duplication using MEGA 7 [44].

\section{Analysis of cis-regulatory elements, intron/exon, and splice variants}

The promoter sequences were retrieved from the genomic sequences as a $1.5 \mathrm{~kb}$ upstream region from the CDS start site of each gene. The (+) and (-) strands of these sequences were analysed using the Plant CARE database for the identification of various cis-regulatory elements [49]. The organization of intron/exon, their numeral, exon length and intron phases in each gene was analysed by aligning the CDS sequence with the respective genomic sequence. The depiction of arrangement was made using the GSDS 2.0 server [50]. The splice variants were analyzed for different sorts of known splicing mechanisms by comparing the variants with their genomic sequence. The existence of these splice variants at transcript level was confirmed by TSA BLAST search.

\section{Physicochemical and structural properties of proteins}

The sequence-dependent properties of TaAPX and TaAPX-R proteins were determined using different servers. The molecular weight, isoelectric point (pl), length, GRAVY value of proteins were determined using ExPasy server and reconfirmed through Ensembl Plants and Sequence Manipulation Suite [42, 51, 52]. The subcellular localization was analyzed using the CELLO v.2.5 [53]. The protein sequences were further screened for the presence of chloroplast transit peptide (cTP) and mitochondrial targeting peptide (mTP) using the ChloroP 1.1 and the TargetP 1.1, respectively [54]. The presence of transmembrane helices was analyzed using the TMHMM v2.0 server [55]. The conserved motifs and domains were analyzed by the MEME Suite [56] and the NCBI conserved domain search [39], respectively.

The multiple sequence alignments were made using the Multalin [57] to examine the primary structure, conserved amino-acid residues, catalytic site, active site, metal-binding sites, and signature motifs. The tertiary structure of proteins was determined by homology modeling through the I-TASSER [58]. The possible ligand-binding site residues were further confirmed using the RaptorX binding site prediction server [59]. The association of organic and inorganic chemicals was analyzed using the STITCH software (http://stitch.embl.de) at high confidence (0.7) with not more than 50 interactions in the first shell. The orthologous proteins of $\mathrm{H}$. vulgare with more than $85 \%$ similarity were considered for the analysis. 


\section{miRNA target prediction}

The targets of known T. aestivum miRNAs [36] in TaAPX and TaAPX-R genes were identified using the psRNATarget server at default parameters [60]. The interactions between the miRNAs and target genes were represented through the Gephi 0.9.1 [61].

\section{Transcriptional profiling and co-expression analyses}

The transcriptional profiling and co-expression analyses of $T a A P X$ and $T a A P X-R$ genes were performed using high throughput RNA-seq data generated from various plant tissues and stress conditions. The fragments per kilobase of transcripts per million mapped reads (FPKM) value was determined through RNA-Seq by Expectation Maximization (RSEM) using Trinity pipeline [62]. The RNA seq data of $T$. aestivum available at wheat-urgi.versailles.inra.fr/files/RNASeqWheat/, developed in two biological replicates from three developmental stages of root, leaf, stem, spike, and grain $[63,64]$ were analyzed for the expression of these genes during development.

The RNA-seq data available in replicates (accession no.- PRJNA243835) after $24 \mathrm{~h}, 48 \mathrm{~h}$ and $72 \mathrm{~h}$ infestation of two major fungal pathogens; Puccinia striiformis (Pst) and Blumeria graminis (Bgt) of T. aestivum [65] were used for expression analysis under biotic stress, separately. The impact of abiotic stresses, heat (HS), drought (DS) and their combination (HD), were studied using RNAseq data (accession no. - SRP045409) available from the leaves in two biological replicates, after one and six hours of treatments, separately [66]. However, the effect of salinity $(150 \mathrm{mM} \mathrm{NaCl})$ stress was analyzed using the RNAseq data (accession no. SRP062745) generated from the root after $6,12,24$ and $48 \mathrm{~h}$ of treatments, separately [67]. The differentially expressed genes ( $\geq 2$ folds) in various treatments were identified using inbuilt edge R software of Trinity pipeline [68]. Heatmaps for differentially expressed genes were constructed using the HCE (Hierarchical Clustering Explorer 3.5) with the Euclidean distance method of the hierarchical clustering of genes [69]. The gene expression was further confirmed using the WheatExp Server (https://wheat.pw.usda.gov/WheatExp/ ).

The co-expression analysis of TaAPXs and TaAPX-Rs with the entire gene pool of T. aestivum during developmental stages and in each stress condition was accomplished using the CoExpressv.1.5 tool. The genes having maximum expression value less than one FPKM were filtered out, and analysis was performed using the Pearson correlation coefficient with correlation power 1 , filtering threshold $\geq 0.9$, bootstraps 100 and false discovery rate (FDR) 10 [70]. The co-expressed genes were annotated and mapped for gene ontology using the Blast2GO software [71]. The interactome was generated for each condition using the Gephi 0.9.1 [61].

\section{Plant samples and growth conditions}

The seeds of $T$. aestivum (cv. Chinese Spring) available in our laboratory [40] were sterilized with $1.2 \%$ sodium hypochlorite and $10 \%$ ethanol. The sterilized seeds were washed with double autoclave water and kept overnight for stratification at $4^{\circ} \mathrm{C}$ on moist Whattman filter papers. The seeds were kept for germination at room temperature. The seedlings were grown in autoclaved water after transferring in fresh phytaboxes and kept in the plant growth chamber. Seven days old seedlings were subjected to different abiotic stresses 
such as heat $\left(40^{\circ} \mathrm{C}\right)$, drought $(20 \% \mathrm{PEG})$ and their combination for 1 and $6 \mathrm{~h}$, while salinity stress $(150 \mathrm{mM}$ $\mathrm{NaCl}$ ) for $6,12,24$ and $48 \mathrm{~h}$, separately. The root and shoot samples of normally grown and stressed plants were immediately frozen in liquid nitrogen, and stored at $-80^{\circ} \mathrm{C}$ for further experimentations.

\section{Expression validation by qRT-PCR}

The stored samples of root and shoot were ground in liquid nitrogen and total RNA was extracted using the SpectrumTM Plant Total RNA kit (Sigma, USA). The DNA contamination was removed using the TURBO DNA-free ${ }^{\mathrm{TM}}$ Kit (Invitrogen, USA). The integrity of isolated RNA samples was checked by gel electrophoresis and quantity was measured using the Nanodrop spectrophotometer. One microgram of RNA was used to synthesize the first-strand cDNA using the Superscript III First Strand Synthesis Supermix (Invitrogen, USA) following the manufacturer's instructions. The gene specific primers were designed for randomly selected $T a A P X$ genes from each phylogenetic group using the Oligo Calc software for qRT-PCR analysis [72]. The qRT-PCR was performed using QuantiFast ${ }^{\circledR}$ SYBR ${ }^{\circledR}$ Green PCR Kit (Qiagen, Germany) on Bio-Rad CFX96 Real-Time PCR detection system following the method established [40]. ADP-ribosylation factor (TaARF1, $A B 050957.1)$ was used as an internal control [73], and fold change in gene expression was calculated using delta-delta CT-method $\left(2^{-\Delta \Delta C T}\right)[74]$.

\section{Determination of ascorbate peroxidase enzyme activity}

For estimation of APX enzyme activity, $0.5 \mathrm{~g}$ of stored plant tissues were ground in liquid nitrogen and homogenized in $50 \mathrm{mM}$ ice cold phosphate buffer ( $\mathrm{pH}$ 7.0) containing $1.0 \mathrm{mM}$ EDTA, followed by centrifugation at $10000 \mathrm{rpm}, 4^{\circ} \mathrm{C}$ for 15 minutes following the previously described methods $[75,76]$ with little modifications. The supernatant was collected and immediately used for the enzyme activity assay. The total protein extracts were quantified according to the method established by Bradford [77] and bovine serum albumin (BSA) was used as standard. The APX activity was determined by analyzing the decrease in absorbance of ascorbate at $290 \mathrm{~nm}$ with an absorbance coefficient of $2.8 \mathrm{mM}^{-1} \mathrm{~cm}^{-1}$ [78] using Eppendorf BioSpectrometer ${ }^{\circledR}$ kinetic. The assay was performed in $1 \mathrm{ml}$ reaction volume consisting of $50 \mathrm{mM}$ phosphate buffer ( $\mathrm{pH} 7.0), 0.5 \mathrm{mM}$ ascorbate, $0.1 \mathrm{mM}$ EDTA, and $0.1 \mathrm{mM}$ hydrogen peroxide. The reaction was started by adding $10 \mu \mathrm{l}$ of crude protein extract and the absorbance decrease was recorded for 3 minutes [78]. All the experiments were performed in triplicates and expressed as mean \pm SD. The APX activity data for heat, drought and their combination was statistically analyzed by analysis of variance (ANOVA) at $5 \%$ probability level using SPSS software. The post hoc Tukey's test was used to examine the significant difference among the treatments $(P<0.05)$. In case of salt stress, the fold change in APX activity was analyzed and expressed as mean $\pm \mathrm{SD}$.

\section{Abbreviations}

APX: Ascorbate peroxidases; Bgt: Blumeria graminis, DS: Drought stress; HD: Heat-drought combination stress; HS: Heat stress; Pst: Puccinia striiformis, ROS: reactive oxygen species

\section{Declarations}




\section{Ethics approval and consent to participate}

Not applicable

\section{Consent for publication}

Not applicable

\section{Availability of data and materials}

All the data have been included in the manuscript. The publically available transcriptome data were used from URGI and NCBI server.

\section{Competing interests}

All the authors declare that there is no competing interest.

\section{Funding}

No funding support.

\section{Authors' contributions}

SKU conceived the idea. SKU and ST designed the experiments. ST and S performed the experiments. ST, PCV and SKU analyzed the data. ST and SKU wrote the manuscript, and ST, KS and SKU finalized the manuscript. All authors have read and approved the manuscript.

\section{Acknowledgements}

Authors are grateful to Panjab University, Chandigarh, India for research facilities, Ensembl Plants, URGI and NCBI for data availability. ST and SKU are grateful to the Science and Engineering Board (SERB), Government of India for Junior Research Fellowship and Early Career Research Award (ECR/2016/001270), respectively.

\section{References}

1. Shigeoka S, Ishikawa T, Tamoi M, Miyagawa Y, Takeda T, Yabuta Y, et al. Regulation and function of ascorbate peroxidase isoenzymes. J Exp Bot. 2002;53:1305-19. doi:10.1093/jexbot/53.372.1305.

2. Boveris A, Sies H, Martino EE, Docampo R, Turrens JF, Stoppani AO. Deficient metabolic utilization of hydrogen peroxide in Trypanosoma cruzi. Biochem J. 1980;188:643-8. https://www.ncbi.nlm.nih.gov/pubmed/7008779.

3. Wada N, Kinoshita S, Matsuo M, Amako K, Miyake C, Asada K. Purification and Molecular Properties of Ascorbate Peroxidase from Bovine Eye. Biochem Biophys Res Commun. 1998;242:256-61. doi:https://doi.org/10.1006/bbrc.1997.7946. 
4. Asada K. THE WATER-WATER CYCLE IN CHLOROPLASTS: Scavenging of Active Oxygens and Dissipation of Excess Photons. Annu Rev Plant Physiol Plant Mol Biol. 1999;50:601-39. doi:10.1146/annurev.arplant.50.1.601.

5. Alscher RG, Erturk N, Heath LS. Role of superoxide dismutases (SODs) in controlling oxidative stress in plants. J Exp Bot. 2002;53:1331-41. doi:10.1093/jexbot/53.372.1331.

6. Jimenez A, Hernandez JA, Del Rio LA, Sevilla F. Evidence for the Presence of the Ascorbate-Glutathione Cycle in Mitochondria and Peroxisomes of Pea Leaves. Plant Physiol. 1997;114:275-84. http://www.ncbi.nlm.nih.gov/pubmed/12223704. Accessed 21 Apr 2017.

7. Teixeira FK, Menezes-Benavente L, Galvão VC, Margis R, Margis-Pinheiro M. Rice ascorbate peroxidase gene family encodes functionally diverse isoforms localized in different subcellular compartments. Planta. 2006;224:300. doi:10.1007/s00425-005-0214-8.

8. Caverzan A, Passaia G, Rosa SB, Ribeiro CW, Lazzarotto F, Margis-Pinheiro M. Plant responses to stresses: Role of ascorbate peroxidase in the antioxidant protection. Genet Mol Biol. 2012;35 4 (suppl):1011-9.

9. Lazzarotto F, Teixeira FK, Rosa SB, Dunand C, Fernandes CL, de Vasconcelos Fontenele A, et al. Ascorbate peroxidase-related (APx-R) is a new heme-containing protein functionally associated with ascorbate peroxidase but evolutionarily divergent. New Phytol. 2011;191:234-50. doi:10.1111/j.14698137.2011.03659.x.

10. Dabrowska G. Characteristics of the plant ascorbate peroxidase family. Acta Biol Cracoviensia Ser Bot. 2007;49:7-17.

11. Lad L, Mewies M, Raven EL. Substrate Binding and Catalytic Mechanism in Ascorbate Peroxidase: Evidence for Two Ascorbate Binding Sites. Biochemistry. 2002;41:13774-81. doi:10.1021/bi0261591.

12. Pnueli L, Liang H, Rozenberg M, Mittler R. Growth suppression, altered stomatal responses, and augmented induction of heat shock proteins in cytosolic ascorbate peroxidase (Apx1)-deficient Arabidopsis plants. Plant J. 2003;34:187-203. http://www.ncbi.nlm.nih.gov/pubmed/12694594. Accessed 7 Dec 2018.

13. Narendra S, Venkataramani S, Shen G, Wang J, Pasapula V, Lin Y, et al. The Arabidopsis ascorbate peroxidase 3 is a peroxisomal membrane-bound antioxidant enzyme and is dispensable for Arabidopsis growth and development. J Exp Bot. 2006;57:3033-42. doi:10.1093/jxb/erl060.

14. Chen C, Letnik I, Hacham Y, Dobrev P, Ben-Daniel B-H, Vanková R, et al. ASCORBATE PEROXIDASE6 Protects Arabidopsis Desiccating and Germinating Seeds from Stress and Mediates Cross Talk between Reactive Oxygen Species, Abscisic Acid, and Auxin. Plant Physiol. 2014;166:370-83. doi:10.1104/pp.114.245324.

15. Kim YJ, Kim S-I, Kesavan M, Kwak JS, Song JT, Seo HS. Ascorbate Peroxidase OsAPx1 is Involved in Seed Development in Rice. Plant Breed Biotechnol. 2015;3:11-20. doi:10.9787/PBB.2015.3.1.011.

16. Zhang Z, Zhang Q, Wu J, Zheng X, Zheng S, Sun X, et al. Gene Knockout Study Reveals That Cytosolic Ascorbate Peroxidase 2(OsAPX2) Plays a Critical Role in Growth and Reproduction in Rice under Drought, Salt and Cold Stresses. PLoS One. 2013;8:e57472. doi:10.1371/journal.pone.0057472. 
17. Lundberg E, Storm P, Schröder W, Funk C. Crystal structure of the TL29 protein from Arabidopsis thaliana: An APX homolog without peroxidase activity. J Struct Biol. 2011;176:24-31.

18. Mullen RT, Trelease RN. The Sorting Signals for Peroxisomal Membrane-bound Ascorbate Peroxidase Are within Its C-terminal Tail. J Biol Chem. 2000;275:16337-44. doi:10.1074/jbc.M001266200.

19. Teixeira FK, Menezes-Benavente L, Margis R, Margis-Pinheiro M. Analysis of the Molecular Evolutionary History of the Ascorbate Peroxidase Gene Family: Inferences from the Rice Genome. J Mol Evol. 2004;59:761-70. doi:10.1007/s00239-004-2666-z.

20. Panchuk II, Zentgraf U, Volkov RA. Expression of the Apx gene family during leaf senescence of Arabidopsis thaliana. Planta. 2005;222:926-32. doi:10.1007/s00425-005-0028-8.

21. Ozyigit II, Filiz E, Vatansever R, Kurtoglu KY, Koc I, Öztürk MX, et al. Identification and Comparative Analysis of H2O2-Scavenging Enzymes (Ascorbate Peroxidase and Glutathione Peroxidase) in Selected Plants Employing Bioinformatics Approaches. Front Plant Sci. 2016;7:301. doi:10.3389/fpls.2016.00301.

22. Tao C, Jin X, Zhu L, Xie Q, Wang X, Li H. Genome-wide investigation and expression profiling of APX gene family in Gossypium hirsutum provide new insights in redox homeostasis maintenance during different fiber development stages. Mol Genet Genomics. 2018;293:685-97. doi:10.1007/s00438-0171413-2.

23. Dunand C, Mathé $C$, Lazzarotto F, Margis R, Margis-Pinheiro M. Ascorbate peroxidase-related (APx-R) is not a duplicable gene. Plant Signal Behav. 2011;6:1908-13. doi:10.4161/psb.6.12.18098.

24. Marcussen T, Sandve SR, Heier L, Spannagl M, Pfeifer M, Jakobsen KS, et al. Ancient hybridizations among the ancestral genomes of bread wheat. Science (80- ). 2014;345:1250092-1250092. doi:10.1126/science.1250092.

25. Tajima F. Simple methods for testing the molecular evolutionary clock hypothesis. Genetics. 1993;135:599-607. http://www.ncbi.nlm.nih.gov/pubmed/8244016. Accessed 21 Aug 2019.

26. Tyagi S, Sharma S, Taneja M, Shumayla, Kumar R, Sembi JK, et al. Superoxide dismutases in bread wheat (Triticum aestivum L.): Comprehensive characterization and expression analysis during development and, biotic and abiotic stresses. Agri Gene. 2017;6.

27. Tyagi S, Himani, Sembi JK, Upadhyay SK. Gene architecture and expression analyses provide insights into the role of glutathione peroxidases (GPXs) in bread wheat (Triticum aestivum L.). J Plant Physiol. 2018;223.

28. Gechev TS, Van Breusegem F, Stone JM, Denev I, Laloi C. Reactive oxygen species as signals that modulate plant stress responses and programmed cell death. BioEssays. 2006;28:1091-101. doi:10.1002/bies.20493.

29. Pandey S, Fartyal D, Agarwal A, Shukla T, James D, Kaul T, et al. Abiotic Stress Tolerance in Plants: Myriad Roles of Ascorbate Peroxidase. Front Plant Sci. 2017;8:581. doi:10.3389/fpls.2017.00581.

30. Park S-Y, Ryu S-H, Jang I-C, Kwon S-Y, Kim J-G, Kwak S-S. Molecular cloning of a cytosolic ascorbate peroxidase cDNA from cell cultures of sweetpotato and its expression in response to stress. Mol Genet Genomics. 2004;271:339-46. doi:10.1007/s00438-004-0986-8. 
31. Shi WM, Muramoto Y, Ueda A, Takabe T. Cloning of peroxisomal ascorbate peroxidase gene from barley and enhanced thermotolerance by overexpressing in Arabidopsis thaliana. Gene. 2001;273:23-7. doi:https://doi.org/10.1016/S0378-1119(01)00566-2.

32. Mishra P, Bhoomika K, Dubey RS. Differential responses of antioxidative defense system to prolonged salinity stress in salt-tolerant and salt-sensitive Indica rice (Oryza sativa L.) seedlings. Protoplasma. 2013;250:3-19. doi:10.1007/s00709-011-0365-3.

33. Hakeem KR, Khan F, Chandna R, Siddiqui TO, Iqbal M. Genotypic Variability Among Soybean Genotypes Under $\mathrm{NaCl}$ Stress and Proteome Analysis of Salt-Tolerant Genotype. Appl Biochem Biotechnol. 2012;168:2309-29. doi:10.1007/s12010-012-9939-6.

34. Turan S, Tripathy BC. Salt and genotype impact on antioxidative enzymes and lipid peroxidation in two rice cultivars during de-etiolation. Protoplasma. 2013;250:209-22. doi:10.1007/s00709-012-0395-5.

35. Kausar R, Hossain Z, Makino T, Komatsu S. Characterization of ascorbate peroxidase in soybean under flooding and drought stresses. Mol Biol Rep. 2012;39:10573-9. doi:10.1007/s11033-012-1945-9.

36. Sun F, Guo G, Du J, Guo W, Peng H, Ni Z, et al. Whole-genome discovery of miRNAs and their targets in wheat (Triticum aestivum L.). BMC Plant Biol. 2014;14:142. doi:10.1186/1471-2229-14-142.

37. Finn RD, Bateman A, Clements J, Coggill P, Eberhardt RY, Eddy SR, et al. Pfam: the protein families database. Nucleic Acids Res. 2014;42:D222-30. doi:10.1093/nar/gkt1223.

38. Fawal N, Li Q, Savelli B, Brette M, Passaia G, Fabre M, et al. PeroxiBase: a database for large-scale evolutionary analysis of peroxidases. Nucleic Acids Res. 2013;41 Database issue:D441-4. doi:10.1093/nar/gks1083.

39. Marchler-Bauer A, Bo Y, Han L, He J, Lanczycki CJ, Lu S, et al. CDD/SPARCLE: functional classification of proteins via subfamily domain architectures. Nucleic Acids Res. 2017;45:D200-3. doi:10.1093/nar/gkw1129.

40. Shumayla P, Sharma S, Pandey AK, Singh K, Upadhyay SK, Zhai W. Molecular Characterization and Global Expression Analysis of Lectin Receptor Kinases in Bread Wheat (Triticum aestivum). PLoS One. 2016;11:e0153925. doi:10.1371/journal.pone.0153925.

41. Taneja M, Upadhyay SK. Molecular characterization and differential expression suggested diverse functions of P-type II Ca2+ATPases in Triticum aestivum L. BMC Genomics. 2018;19:389. doi:10.1186/s12864-018-4792-9.

42. Nussbaumer T, Martis MM, Roessner SK, Pfeifer M, Bader KC, Sharma S, et al. MIPS PlantsDB: a database framework for comparative plant genome research. Nucleic Acids Res. 2013;41 Database issue:D1144-51. doi:10.1093/nar/gks1153.

43. Edgar RC. MUSCLE: multiple sequence alignment with high accuracy and high throughput. Nucleic Acids Res. 2004;32:1792-7. doi:10.1093/nar/gkh340.

44. Kumar S, Stecher G, Tamura K. MEGA7: Molecular Evolutionary Genetics Analysis Version 7.0 for Bigger Datasets. Mol Biol Evol. 2016;33:1870-4. doi:10.1093/molbev/msw054.

45. Krzywinski M, Schein J, Birol I, Connors J, Gascoyne R, Horsman D, et al. Circos: an information aesthetic for comparative genomics. Genome Res. 2009;19:1639-45. doi:10.1101/gr.092759.109. 
46. Zhang Z, Xiao J, Wu J, Zhang H, Liu G, Wang X, et al. ParaAT: A parallel tool for constructing multiple protein-coding DNA alignments. Biochem Biophys Res Commun. 2012;419:779-81.

doi:https://doi.org/10.1016/j.bbrc.2012.02.101.

47. Wang D-P, Wan H-L, Zhang S, Yu J. \$y\$-MYN: a new algorithm for estimating Ka and Ks with consideration of variable substitution rates. Biol Direct. 2009;4:20. doi:10.1186/1745-6150-4-20.

48. Gaut BS, Morton BR, McCaig BC, Clegg M. Substitution rate comparisons between grasses and palms: Synonymous rate differences at the nuclear gene Adh parallel rate differences at the plastid gene rbcL. 1996.

49. Lescot M, Déhais P, Thijs G, Marchal K, Moreau Y, Van de Peer Y, et al. PlantCARE, a database of plant cis-acting regulatory elements and a portal to tools for in silico analysis of promoter sequences. Nucleic Acids Res. 2002;30:325-7. http://www.ncbi.nlm.nih.gov/pubmed/11752327. Accessed 21 Apr 2017.

50. Hu B, Jin J, Guo A-Y, Zhang H, Luo J, Gao G. GSDS 2.0: an upgraded gene feature visualization server. Bioinformatics. 2015;31:1296-7. doi:10.1093/bioinformatics/btu817.

51. Gasteiger E, Hoogland C, Gattiker A, Duvaud S, Wilkins MR, Appel RD, et al. Protein Identification and Analysis Tools on the ExPASy Server. 2018. p. 571-607. doi:10.1385/1-59259-890-0:571.

52. Stothard P. The Sequence Manipulation Suite: JavaScript Programs for Analyzing and Formatting Protein and DNA Sequences. Biotechniques. 2000;28:1102-4. doi:10.2144/00286ir01.

53. Yu C-S, Chen Y-C, Lu C-H, Hwang J-K. Prediction of protein subcellular localization. Proteins Struct Funct Bioinforma. 2006;64:643-51. doi:10.1002/prot.21018.

54. Emanuelsson O, Nielsen H, Brunak S, Heijne G. Predicting Subcellular Localization of Proteins Based on their N-terminal Amino Acid Sequence. 2000.

55. Krogh A, Larsson B, von Heijne G, Sonnhammer EL. Predicting transmembrane protein topology with a hidden markov model: application to complete genomes11Edited by F. Cohen. J Mol Biol. 2001;305:567-80. doi:10.1006/jmbi.2000.4315.

56. Bailey TL, Boden M, Buske FA, Frith M, Grant CE, Clementi L, et al. MEME SUITE: tools for motif discovery and searching. Nucleic Acids Res. 2009;37 Web Server:W202-8. doi:10.1093/nar/gkp335.

57. Corpet F. Multiple sequence alignment with hierarchical clustering. Nucleic Acids Res. 1988;16:1088190. https://www.ncbi.nlm.nih.gov/pubmed/2849754.

58. Zhang Y. I-TASSER server for protein 3D structure prediction. BMC Bioinformatics. 2008;9:40. doi:10.1186/1471-2105-9-40.

59. Wang S, Li W, Liu S, Xu J. RaptorX-Property: a web server for protein structure property prediction. Nucleic Acids Res. 2016;44:W430-5. doi:10.1093/nar/gkw306.

60. Dai X, Zhao PX. psRNATarget: a plant small RNA target analysis server. Nucleic Acids Res. 2011;39 Web Server issue:W155-9. doi:10.1093/nar/gkr319.

61. Bastian M, Heymann S, Jacomy M. Gephi: An Open Source Software for Exploring and Manipulating Networks. 2009.

62. Haas BJ, Papanicolaou A, Yassour M, Grabherr M, Blood PD, Bowden J, et al. De novo transcript sequence reconstruction from RNA-seq using the Trinity platform for reference generation and analysis. Nat Protoc. 2013;8:1494-512. doi:10.1038/nprot.2013.084. 
63. Choulet F, Alberti A, Theil S, Glover N, Barbe V, Daron J, et al. Structural and functional partitioning of bread wheat chromosome 3B. Science (80- ). 2014;345:1249721-1249721.

doi:10.1126/science.1249721.

64. Pingault L, Choulet F, Alberti A, Glover N, Wincker P, Feuillet C, et al. Deep transcriptome sequencing provides new insights into the structural and functional organization of the wheat genome. Genome Biol. 2015;16:29. doi:10.1186/s13059-015-0601-9.

65. Zhang H, Yang Y, Wang C, Liu M, Li H, Fu Y, et al. Large-scale transcriptome comparison reveals distinct gene activations in wheat responding to stripe rust and powdery mildew. BMC Genomics. 2014;15:898. doi:10.1186/1471-2164-15-898.

66. Liu Z, Xin M, Qin J, Peng H, Ni Z, Yao Y, et al. Temporal transcriptome profiling reveals expression partitioning of homeologous genes contributing to heat and drought acclimation in wheat (Triticum aestivum L.). BMC Plant Biol. 2015;15:152. doi:10.1186/s12870-015-0511-8.

67. Zhang Y, Liu Z, Khan AA, Lin Q, Han Y, Mu P, et al. Expression partitioning of homeologs and tandem duplications contribute to salt tolerance in wheat (Triticum aestivum L.). Sci Rep. 2016;6:21476. doi:10.1038/srep21476.

68. Haas BJ, Papanicolaou A, Yassour M, Grabherr M, Blood PD, Bowden J, et al. De novo transcript sequence reconstruction from RNA-seq using the Trinity platform for reference generation and analysis. Nat Protoc. 2013;8:1494-512. doi:10.1038/nprot.2013.084.

69. Seo J, Gordish-Dressman H, Hoffman EP. An interactive power analysis tool for microarray hypothesis testing and generation. Bioinformatics. 2006;22:808-14. http://dx.doi.org/10.1093/bioinformatics/btk052.

70. Nazarov P, Muller A, Khutko V, Vallar L. Co-Expression Analysis of Large Microarray Data Sets Using CoExpress Software Tool. 2010.

71. Conesa A, Götz S. Blast2GO: A Comprehensive Suite for Functional Analysis in Plant Genomics. 2008.

72. Kibbe WA. OligoCalc: an online oligonucleotide properties calculator. Nucleic Acids Res. 2007;35 Web Server:W43-6. doi:10.1093/nar/gkm234.

73. Shumayla, Tyagi S, Sharma A, Singh K, Upadhyay SK. Genomic dissection and transcriptional profiling of Cysteine-rich receptor-like kinases in five cereals and functional characterization of TaCRK68-A. Int J Biol Macromol. 2019;134:316-29. doi:10.1016/j.ijbiomac.2019.05.016.

74. Livak KJ, Schmittgen TD. Analysis of Relative Gene Expression Data Using Real-Time Quantitative PCR and the 2- $\Delta \Delta C T$ Method. Methods. 2001;25:402-8. doi:10.1006/meth.2001.1262.

75. Zhang B, Chu G, Wei C, Ye J, Li Z, Liang Y. The growth and antioxidant defense responses of wheat seedlings to omethoate stress. Pestic Biochem Physiol. 2011;100:273-9.

76. Gietler M, Nykiel M, Zagdańska BM. Changes in the reduction state of ascorbate and glutathione, protein oxidation and hydrolysis leading to the development of dehydration intolerance in Triticum aestivum $\mathrm{L}$. seedlings. Plant Growth Regul. 2016;79:287-97. doi:10.1007/s10725-015-0133-z.

77. Bradford MM. A rapid and sensitive method for the quantitation of microgram quantities of protein utilizing the principle of protein-dye binding. Anal Biochem. 1976;72:248-54. doi:10.1016/00032697(76)90527-3. 
78. Nakano Y, Asada K. Hydrogen Peroxide is Scavenged by Ascorbate-specific Peroxidase in Spinach Chloroplasts. Plant Cell Physiol. 1981;22:867-80. doi:10.1093/oxfordjournals.pcp.a076232.

\section{Tables}

Table 1 The ratio of $\mathrm{Ka} / \mathrm{Ks}$ between duplicated TaAPX gene pairs and their divergence time.

\begin{tabular}{lllllll}
\hline Gene A & Gene B & Ka & Ks & Ka/Ks & T = Ks/2r & Selection pressure \\
\hline TaAPX2-A & TaAPX5-A & 0.097633 & 1.03639 & 0.0942044 & 7.972230769 & Purifying selection \\
TaAPX2-B & TaAPX5-B & 0.095283 & 1.20929 & 0.0787927 & 9.302230769 & Purifying selection \\
TaAPX2-D & TaAPX5-D & 0.101729 & 1.17689 & 0.0864383 & 9.053 & Purifying selection \\
\hline
\end{tabular}

Ka- non-synonymous substitutions per non-synonymous site, Ks- synonymous substitutions per synonymous site, $\mathrm{T}$ - Divergence time

Table 2 Tajima's relative rate test for duplicated TaAPX gene pairs.

\begin{tabular}{llllllll}
\hline Gene A & Gene B & Out-group & Nt & Na & Nb & $\chi^{\square}$ & $P$ \\
\hline TaAPX2-A & TaAPX5-A.1 & OsAPX1 & 527 & 87 & 28 & 30.27 & $<0.05$ \\
TaAPX2-B & TaAPX5-B.1 & OsAPX1 & 578 & 103 & 27 & 44.43 & $<0.05$ \\
TaAPX2-D & TaAPX5-D & OsAPX1 & 575 & 104 & 29 & 42.29 & $<0.05$ \\
\hline
\end{tabular}

Nt Identical sites in all three sequences

$\mathrm{Na} \quad$ Unique differences in Sequence A

$\mathrm{Nb} \quad$ Unique differences in Sequence B

If $P<0.05 \quad \chi \square$ value is significant and the test rejects the hypothesis of equal rates of substitution between the duplicated genes suggesting that they have different evolutionary rates after divergence

Table 3 Molecular characterization of TaAPX genes and proteins. 


\begin{tabular}{|c|c|c|c|c|c|}
\hline Gene & Chromosome & ORF (bp) & Protein length & Isoelectric Point & Molecular Weight (kDa) \\
\hline TaAPX1-A & $2 \mathrm{~A}$ & 888 & 295 & 8.31 & 32.36 \\
\hline ТаAPX1-B & $2 \mathrm{~B}$ & 894 & 297 & 8.30 & 32.38 \\
\hline TaAPX1-D & $2 \mathrm{D}$ & 888 & 295 & 8.30 & 32.35 \\
\hline ТаAPX2-A & $2 \mathrm{~A}$ & 1227 & 408 & 6.95 & 44.33 \\
\hline Та $A P X 2-B$ & $2 \mathrm{~B}$ & 765 & 254 & 5.00 & 27.43 \\
\hline TaAPX2-D & $2 \mathrm{D}$ & 771 & 256 & 4.89 & 27.66 \\
\hline ТаАРХЗ-А. 1 & $2 \mathrm{~A}$ & 1038 & 345 & 8.72 & 37.19 \\
\hline ТаАРХЗ-А. 2 & $2 \mathrm{~A}$ & 1041 & 346 & 8.42 & 37.32 \\
\hline ТаАРХЗ-В & $2 \mathrm{~B}$ & 1047 & 348 & 8.72 & 37.51 \\
\hline ТаAPXЗ-D & $2 \mathrm{D}$ & 1047 & 348 & 8.72 & 37.54 \\
\hline ТаAPX4-A & $2 \mathrm{~A}$ & 1059 & 352 & 7.76 & 37.77 \\
\hline ТаAPX4-B & $2 \mathrm{~B}$ & 1134 & 377 & 9.25 & 40.63 \\
\hline TaAPX4-D & $2 \mathrm{D}$ & 1140 & 379 & 9.07 & 40.74 \\
\hline ТаАРХ5-А. 1 & $4 \mathrm{~A}$ & 684 & 227 & 7.15 & 24.77 \\
\hline ТаAPХ5-A.2 & $4 \mathrm{~A}$ & 753 & 250 & 6.17 & 27.49 \\
\hline ТаАРХ5-В. 1 & $4 \mathrm{~B}$ & 753 & 250 & 6.17 & 27.47 \\
\hline ТаАРХ5-В.2 & $4 \mathrm{~B}$ & 771 & 256 & 7.60 & 27.90 \\
\hline TаAPX5-D & $4 \mathrm{D}$ & 753 & 250 & 6.32 & 27.49 \\
\hline ТаАРХ6-А. 1 & $5 \mathrm{~A}$ & 939 & 312 & 7.02 & 34.12 \\
\hline ТаAPХ6-A.2 & $5 \mathrm{~A}$ & 936 & 311 & 7.30 & 33.99 \\
\hline ТаАРХ & $5 \mathrm{~A}$ & 921 & 306 & 6.80 & 33.38 \\
\hline ТаАРХб-В. 1 & $5 B$ & 924 & 307 & 7.06 & 33.57 \\
\hline ТаАРХб-В.2 & $5 B$ & 939 & 312 & 7.66 & 34.18 \\
\hline ТаАРХ6-В.3 & $5 B$ & 942 & 313 & 7.32 & 34.31 \\
\hline ТаAPХ6-D.1 & $5 \mathrm{D}$ & 924 & 307 & 6.61 & 33.44 \\
\hline TаAPX6-D.2 & $5 \mathrm{D}$ & 942 & 313 & 6.61 & 34.21 \\
\hline ТаAPX6-D.3 & $5 \mathrm{D}$ & 936 & 311 & 6.61 & 34.05 \\
\hline ТаAPX7-A & $6 \mathrm{~A}$ & 1296 & 431 & 6.80 & 46.86 \\
\hline ТаАРХ7-В.1 & $6 \mathrm{~B}$ & 1296 & 431 & 7.58 & 46.75 \\
\hline ТаАРХ7-В.2 & $6 B$ & 1320 & 439 & 8.04 & 47.65 \\
\hline ТаАРХ7-В.3 & $6 \mathrm{~B}$ & 1410 & 469 & 8.61 & 50.90 \\
\hline TаAPX7-D.1 & $6 \mathrm{D}$ & 1296 & 431 & 7.13 & 46.80 \\
\hline TаAPX7-D.2 & $6 \mathrm{D}$ & 1266 & 421 & 7.58 & 45.57 \\
\hline ТаАРХ $8-A .1$ & $7 \mathrm{~A}$ & 696 & 231 & 8.21 & 24.60 \\
\hline ТаAРХ8-A.2 & $7 \mathrm{~A}$ & 990 & 329 & 7.00 & 35.58 \\
\hline ТаАРХ8-В. 1 & $7 B$ & 1026 & 341 & 7.38 & 36.52 \\
\hline ТаАРХ8-B.2 & $7 B$ & 1002 & 333 & 6.99 & 35.67 \\
\hline ТаAPX8-D.1 & $7 \mathrm{D}$ & 987 & 328 & 6.05 & 35.12 \\
\hline ТаAPX8-D.2 & $7 \mathrm{D}$ & 744 & 247 & 4.98 & 26.48 \\
\hline ТаАРХЯ-А & $7 \mathrm{~A}$ & 876 & 291 & 8.19 & 31.67 \\
\hline ТаАРХ9-В & $7 B$ & 876 & 291 & 8.19 & 31.72 \\
\hline TаAPX9-D.1 & $7 \mathrm{D}$ & 876 & 291 & 8.19 & 31.68 \\
\hline ТаAPX9-D.2 & $7 \mathrm{D}$ & 777 & 258 & 8.43 & 28.55 \\
\hline
\end{tabular}

Table 4 Details of predicted tertiary structures and ligand binding sites in different APX proteins. 


\begin{tabular}{|c|c|c|c|c|c|c|c|c|}
\hline \multicolumn{2}{|c|}{ Protein details } & \multicolumn{3}{|c|}{ Structural parameters } & \multicolumn{4}{|c|}{ Ligand and binding site residues } \\
\hline Protein & $\begin{array}{l}\text { Sub-cellular } \\
\text { localization }\end{array}$ & $\begin{array}{l}\text { C- } \\
\text { score }\end{array}$ & TM-score & RMSD & Ligand & $\begin{array}{l}\mathrm{P}- \\
\text { value }\end{array}$ & Multiplicity & $\begin{array}{l}\text { Binding } \\
\text { residues }\end{array}$ \\
\hline $\begin{array}{l}\text { TaAPX1- } \\
\text { A }\end{array}$ & Peroxisome & -1.55 & $0.52 \pm 0.15$ & $9.7 \pm 4.6 \AA$ & HEM & $\begin{array}{l}4.25 \mathrm{e}- \\
09\end{array}$ & 131 & $\begin{array}{l}\text { P35 I36 } \\
\text { L38 } \\
\text { R39 } \\
\text { W42 } \\
\text { P133 } \\
\text { D134 } \\
\text { A135 } \\
\text { L142 } \\
\text { F146 } \\
\text { L159 } \\
\text { S160 } \\
\text { G162 } \\
\text { H163 } \\
\text { L165 } \\
\text { G166 } \\
\text { K167 } \\
\text { A168 } \\
\text { R169 } \\
\text { R172 } \\
\text { S173 } \\
\text { W179 } \\
\text { L205 } \\
\text { T207 }\end{array}$ \\
\hline $\begin{array}{l}\text { TaAPX2- } \\
\text { B }\end{array}$ & Cytoplasm & 1.60 & $0.94 \pm 0.05$ & $2.7 \pm 2.0 \AA$ & HEM & $\begin{array}{l}4.69 \mathrm{e}- \\
10\end{array}$ & 121 & $\begin{array}{l}\text { P35 } \\
\text { L36 } \\
\text { L38 } \\
\text { R39 } \\
\text { W42 } \\
\text { P133 } \\
\text { D134 } \\
\text { A135 } \\
\text { L142 } \\
\text { F146 } \\
\text { L160 } \\
\text { S161 } \\
\text { G163 } \\
\text { H164 } \\
\text { L166 } \\
\text { G167 } \\
\text { R168 } \\
\text { C169 } \\
\text { H170 } \\
\text { R173 } \\
\text { S174 } \\
\text { W180 } \\
\text { L206 } \\
\text { T208 }\end{array}$ \\
\hline $\begin{array}{l}\text { TaAPX3- } \\
\text { A.1 }\end{array}$ & Chloroplast & -0.27 & $0.68 \pm 0.12$ & $7.1 \pm 4.1 \AA$ & HEM & $\begin{array}{l}4.24 \mathrm{e}^{-} \\
12\end{array}$ & 125 & $\begin{array}{l}\text { P97 } \\
\text { V100 } \\
\text { R101 } \\
\text { W104 } \\
\text { P202 } \\
\text { D203 } \\
\text { A204 } \\
\text { L213 } \\
\text { F217 }\end{array}$ \\
\hline
\end{tabular}


TaAPX8- Chloroplast 0.38 B. 1

$0.76 \pm 0.10$

5.7 $\pm 3.6 \AA \quad H E M$

$3.31 \mathrm{e}-155$

08

T293

A121

G122

L124

R125

F128

P220

E221

E222

L229

F233

R247

Q249

L254

S255

A257

H258

I260

G261

G262

K263

I292

L294

T296

D297

C-score - Confidence score of predicted models and ranged between -5 and 2 , where higher value signifies a model with more confidence.

TM-score and RMSD - For quality structure prediction based on the correlation with C-score, where $>0.5$ TM-score directs a model of precise topology.

Multiplicity - For determining the quality of predicted pocket for ligand binding, where value $>40$ indicates the higher confidence.

\section{Additional File Legends}

Additional file 1 Homeologous gene clustering based on $\geq 90 \%$ sequence identity and localized on same chromosome of each A, B and D sub-genomes. Nomenclature assigned in accordance with the guidelines of international rules of genetic nomenclature for gene symbolization in wheat. 
Additional file 2 Molecular characterization of TaAPX genes and proteins of Triticum aestivum and their various physico-chemical properties.

Additional file 3 Existence of transcripts confirmed using BLASTn search against transcriptome shotgun assembly (TSA) sequences of $T$. aestivum at NCBI server.

Additional file 4 Co-expression analysis, and gene ontology (GO) mapping and annotation of co-expressed genes with TaAPXs in tissue developmental stages and during biotic, salt, and heat, drought and their combination stresses.

Additional file 5 Identified target TaAPXs of known miRNAs in T. aestivum using psRNATarget.

Additional file 6 Annotation of chemical compounds interact with TaAPXs.

Additional table 1 Cis-regulatory elements predicted in promoter region of TaAPX genes.

Additional table 2 List of qRT PCR gene specific primers.

Additional fig. 1 Sequences of ten conserved motifs indicating the motif number, e-value, sites, motif width.

Additional fig. 2 Expression analysis using qRT PCR of few randomly selected TaAPX genes. qRT PCR was performed using gene specific primers. The bars represents the $\log _{2}$ fold change expression value under different abiotic stress conditions and vertical lines on bars represent standard deviation. The fold change value of each gene under heat (HS), drought (DS) and their combination (HD) are shown in graphs (A-D), whereas $(\mathrm{E}-\mathrm{H})$ represents fold change value under salt stress. The result shows the expression of each $T a A P X$ gene was comparable to the expression obtained from RNA seq data.

\section{Figures}




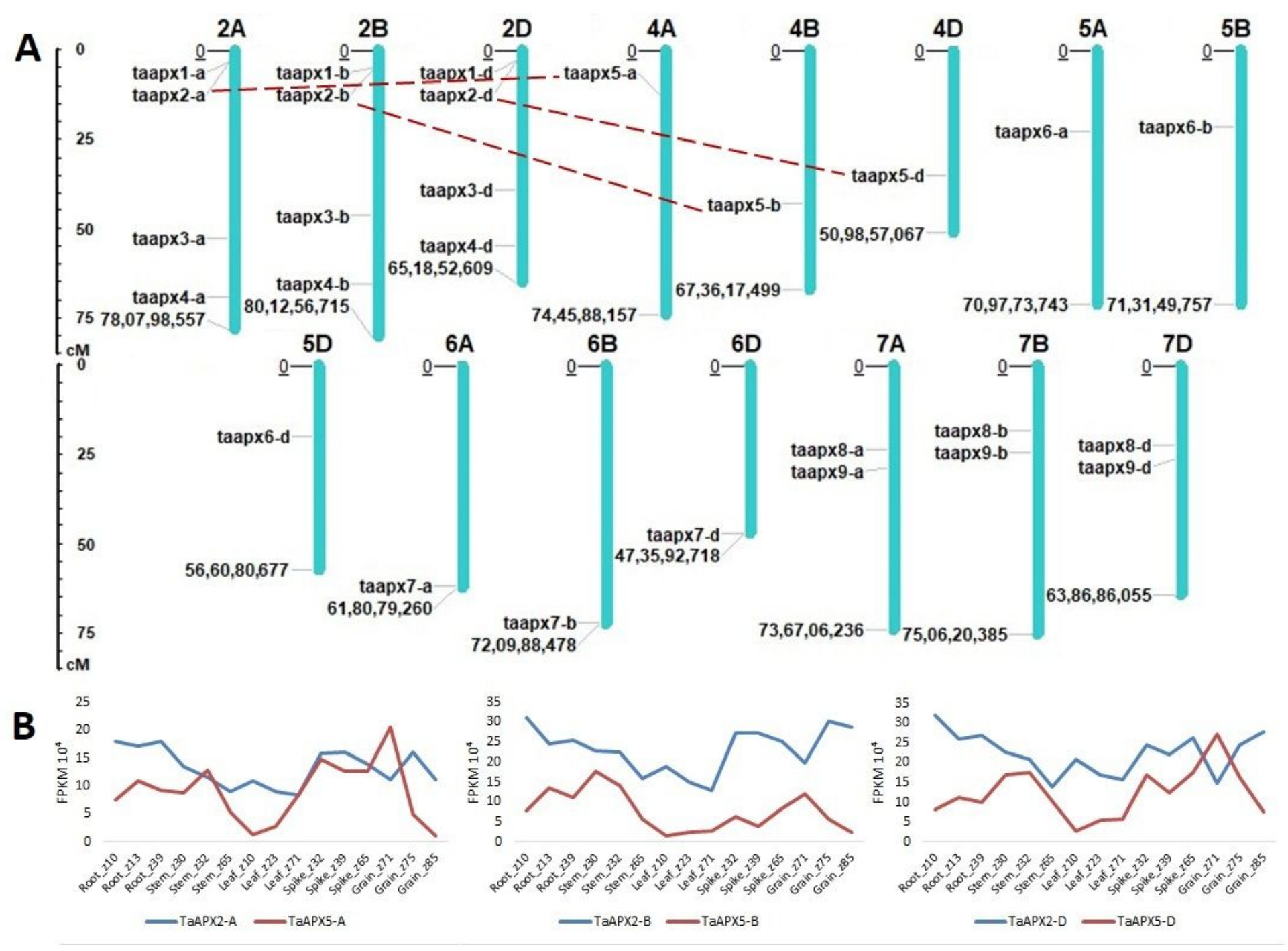

\section{Figure 1}

Chromosomal localization of TaAPX genes and expression profiles of paralogous genes. Localization of TaAPX genes on various chromosomes of $A, B$ and $D$ sub-genomes. Paralogous gene pairs (TaAPX2-A \&TaAPX5-A, TaAPX2-A \&TaAPX5-A and TaAPX2-A \&TaAPX5-A) are shown with red dashed lines (a). The expression profiles of paralogous gene pairs in three developmental stages of each root, leaf, stem, spike and grain tissue, indicating the neo-functionalization after duplication (b). 

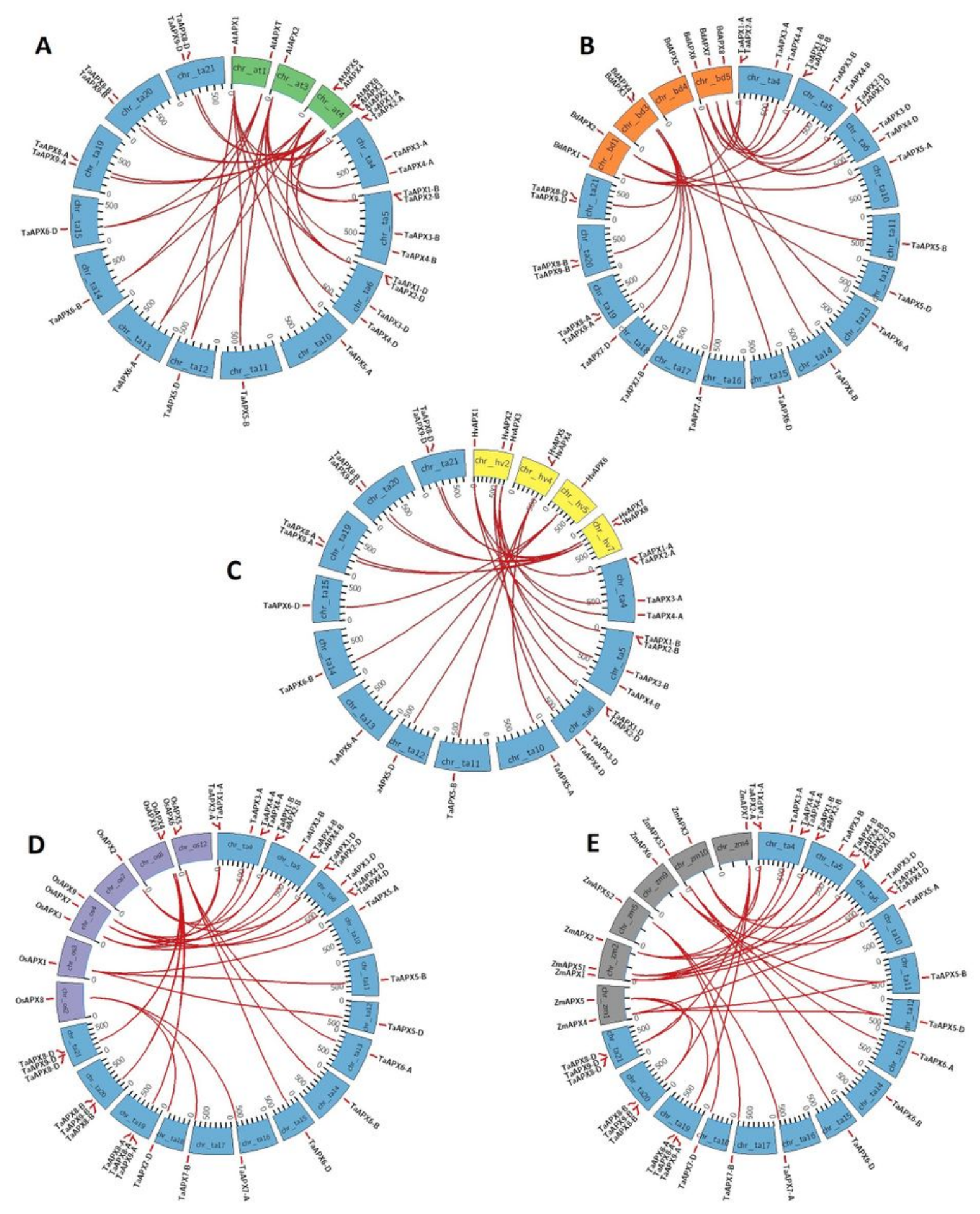

\section{Figure 2}

Synteny analysis. Syntenic relationship of TaAPXs with the APXs of Arabidopsis thaliana (a), Brachypodium distachyon(b), Hordeum vulgare (c), Oryza sativa (d) and Zea mays (e). The figure was generated using circos-0.69-6 software. 


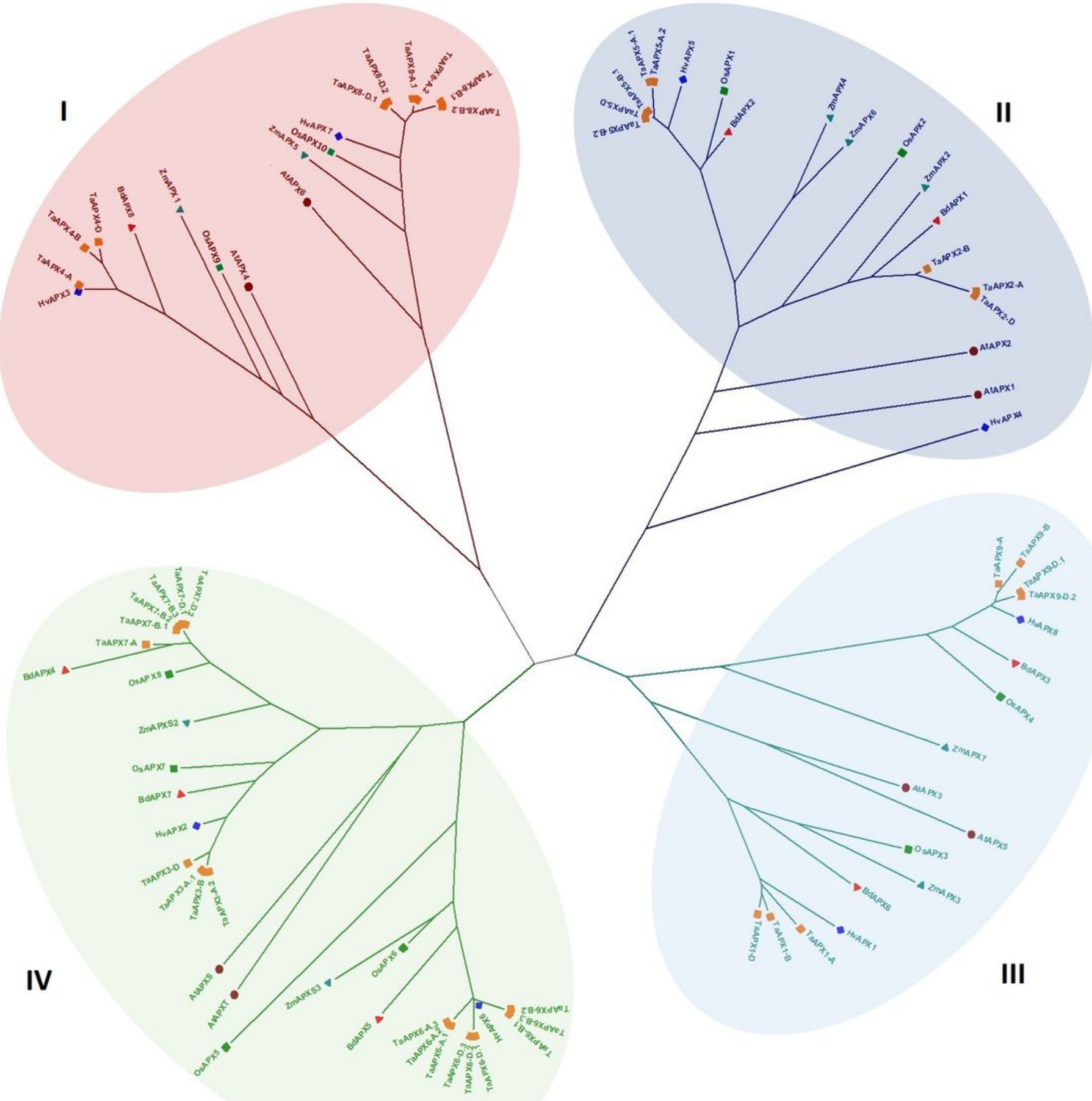

\section{Figure 3}

Phylogenetic analysis. TaAPX proteins along with the APXs of Arabidopsis thaliana, Brachypodium distachyon, Hordeum vulgare, Oryza sativa, and Zea mays were used for the phylogeny construction. Clade I, II, III and IV represents the APX related, chloroplastic/mitochondrial, peroxisomal and cytoplasmic APX proteins, shaded with pink, blue, light blue and green colour, respectively. 
A

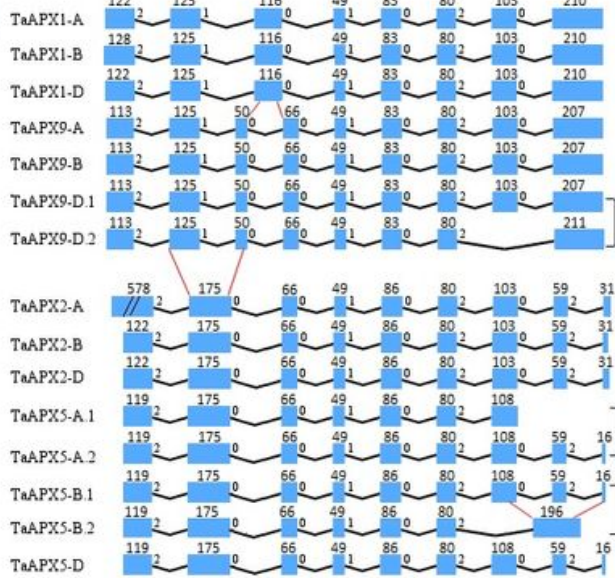

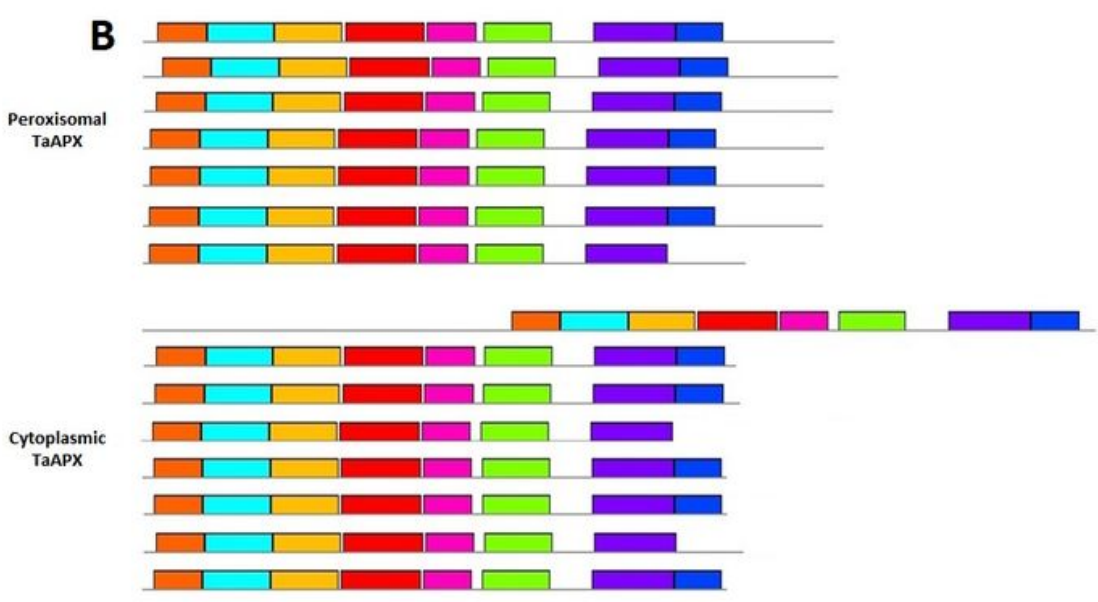

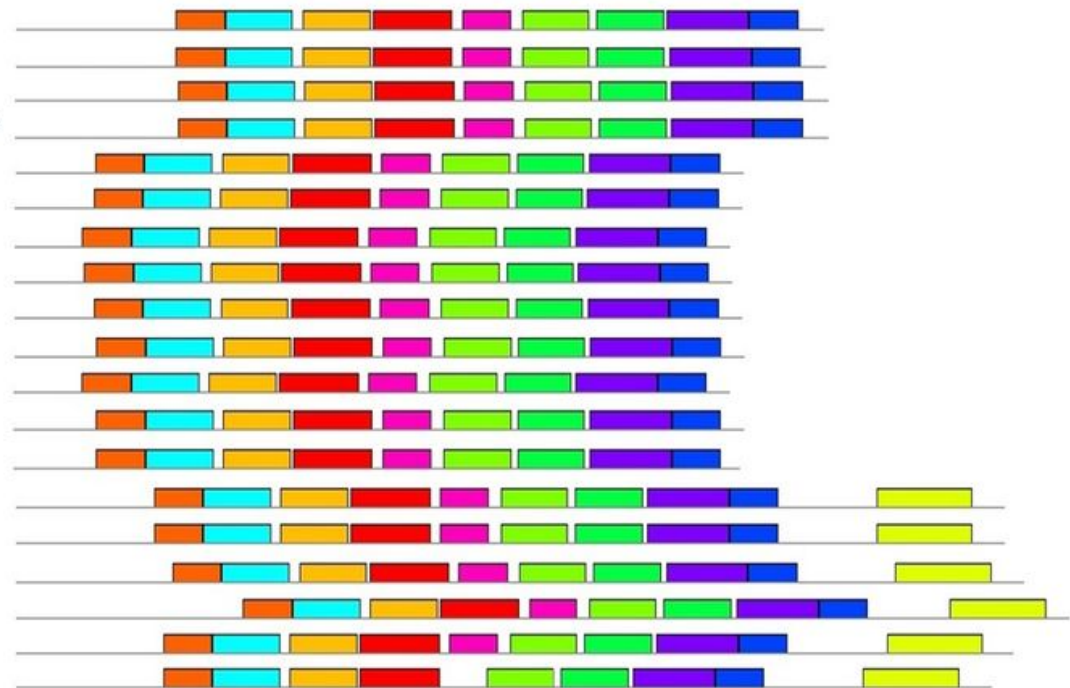

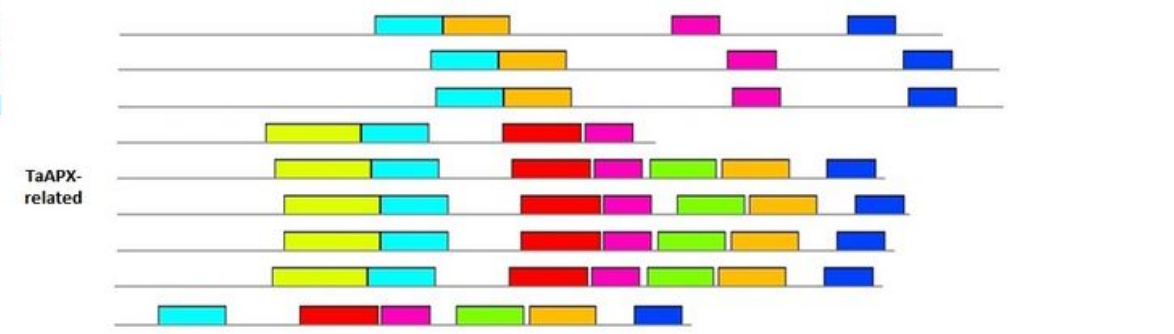

Motif 1 Motif 2 Motif 3 Motif 4 Motif 5 Motif 6 Motif 7 Motif 8 Motif 9 Motif 10

Legend

CDS $\sim$ Intron 012 intron phuse

\section{Figure 4}

Exon/Intron organization and motif analysis. Exon/Intron organization in TaAPX genes and their splice variants. The exons are shown as blue boxes interspaced by introns shown as thin black line. Splice variants are marked with black vertical line at $3^{\prime}$ end of the transcript. The length of each exon is represented above the respective exon. 0, 1 and 2 indicates the different intron phases (a). Motifs analysis showing the organization of ten conserved motifs represented by colored boxes (b). 
A

TaAPX2-A

TaAPX2-D

TaAPX2-B

PsfPX1

TafPX5-A.1

TaAPX5-A.2

TaAPX5-B.1

TaAPX5-D

TaAPX5-B.2

B

TaAPX1-A

TaAPX1-D

TaAPX1-B

TafPX9-A

TaAPX9-B

TaAPX9-D.1

TafPX9-D.2

At $A P X 5$

C

TafPX3-A.1

TaAPX3-A.2

TaAPX3-B

TaAPX3-D

TaAPX7-f

TaAPX7-B.1

TaAPX7-0.1

TaAPX7-B

TaAPX7-B.3

TaAPX7-0.2

At.PPXT

AtAPXS

TaAPX6-A, 1

TaAPX6-P.?

TaAPX6-B.?

TaAPX6-B.3

TaAPX6-D. 2

TaAPX6-D.

TafPX6-A.3

TaAPX6-B.1

TaAPX6-D.1

D

TafPX4-A

TaAPX4-B

TaAPX4-D

At. $P$ P 4

TaAPX8-A.1

TaAPX8-P.2

TaAPX8-D.1

TaAPX8-B.1

TaAPX8-B.?

TaAPX8-D.2
30 ASD 40

AEKNCAPLHLRLAMHSAGTF

AEKNCAPLMLRLAHHSAGTF

AEKNCAPLHLRLAHHSAGTF

AEKKCAPLILRLAMHSAGTF

AEKNCSPLYLRLALHSAGTF

AEKNCSPLHLRLAHHSAGTF

AEKNCSPLHLRLAMHSAGTF

AEKNCSPLMLRLAHHSAGTF

AEKNCSPLHLRLALHSAGTF

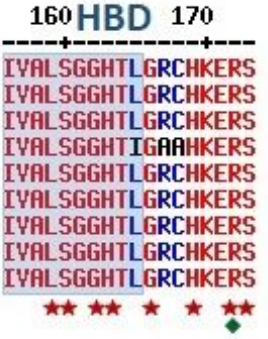

\begin{tabular}{lr}
\hline 180 CBD 190 & 250 \\
\hline ATANPLIFDNSYF & LGFGEA \\
\hline HTANPLIFDNSYF & LGFGEA \\
\hline HTANPLIFDNSYF & LGFGEA \\
\hline HTSNPLIFDNSYF & LGFAEA \\
\hline HTRNPLKFDNSYF & \\
\hline HTRNPLKFDNSYF & LGYAEA \\
\hline HTRNPLKFDNSYF & LGYAEA \\
\hline HTRNPLKFDHSYF & LGYAEA \\
\hline HTRNPLKFDNSYF & HLIIIL \\
\hline &
\end{tabular}

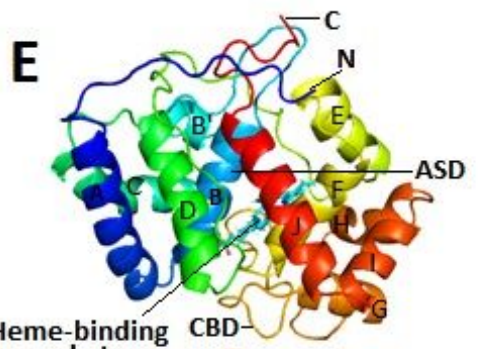

..

.

p

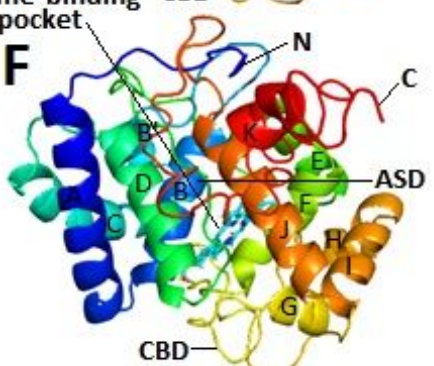

131140 ASD $150 \mathrm{Chl}-\mathrm{D} 1$

I--THCHPILYRLGHHDSGTYDKNIKDHP NTTHCHPIL YRL GHHDSGTYDKNIKDAP NTTHCHPILYRLGHHDSGTYDKNIKDHP NTTHCHPIL YRLGHHDSG TYDKNINDHP KTTYCHPILYRLGHHDSG TYDKHIEEAP KTTYCHPILYRLGHHDSGTYDKNIEEMP KTTYCHPILYRLGHHDSGTYDKNIEEMP KTTYCHPTL YRL GHHDSG TYDKNTEEHP KTTYCHPILYRLGHHDSGTYDIKNIEEMP KTTYCHPILYRLGHHDSGTYDKNIEEAP RTKFCHPILYRLGHHDAGTYNKNIEEMP STKFCHPILYRLGHHDAGTYNKMIKEMP KEKSCHPIL YRL GHHDAG TYDKNIHEMP KEKSCHPIL YRLGHHDAGTYDKNINEMP KEKSCHPIL YRLGHHDAG TYDKNISEUP KEKSCHPILYRLGHHDAGTYDKHISEHP KEKSCHPIL YRL GLHDAGTYDKNISEHP KEKSCHPIL YRL GHHDAGTYDKNISEMP KEKSCHPILYRLGHHDAG TYDKNINEHP KEKSCHPIL YRLGHHDAGTYDKNISEMP

KEKSCHPIL YRLGHHDAG TYDKNISEMP

$\bullet \bullet \star \star \star \star$

$140 \quad 150$ ASD 160

ARIE FKPELYPSLLTLALNDAMTYOK AAIEPKPEL UPSLLTLALHDATIYDK ARIEARPELYPSLLTLALNDAMTYOK TIIKAKPEL YPSLLKLALNDAHTYDK KYKAF------GYLRLYFHDAGTFDI KYKA $9------G Y L R L$ YFHDAGTFDI KYKA KYKAF------GYLRLYFHDAGTFDI

KYKAF------GYLRLYFHDAGTFDI

KYKA

$290 \mathrm{Chl}-\mathrm{D} 2300$

310 CBD

IYHLSGAHTLGRSRPERSGHGKPETKYTKMGPGAPGGQS MTHEMLKFDNSYA IYALSGHHTLGRSRPERSGHGKPETKYTKNGPGAPGGQS HTHEMLKFDNSYF IUAL SGPHTL GRSRPERSGHGKPETKYTKMGPG GPGGOSHTAEUL KFDNSYF IYALSGAHTL GRSRPERSGHGKPETKYTKNGPGAPGGQSHTAEHLKFDNSYP IYALSGAHTL GRSRPDRSGHGKPETKYTKDGPGEPGGQSHTAEHLKFDNSYP IYALSGAHTL GRSRPDRSGHGKPETKYTKDGPGEPGGQSHTAEHLKFDNSYF IYALSGAHTL GRSRPDRSGHGKPETKYTKDGPGEPGGQS MTAEHLKFDNSYF TUALSGAHTL GRSRPDRSGHGKPETKYTKDGPGEPGGOSHTAEHLKFDNSYA IYALSGPHTL GRSRPDRSGHGKPETKYTKDGPGEPGGQSHTREHLKFDNSYP IYALSGAHTLGRSRPDRSGHGKPETKYTKDGPGEPGGQSHTAEMLKFDNSYP IYALSGAHTL GRARPDRSGHGKPETKYTKTGPGEAGGQSHTYKLLKFDNSYF IYALSGAHTL GRSRPERSGHGKPETKYTKEGPGAPGGQS MTPEHLKFDNSYF IYALSGPHTL GRSRPERSGHGKPETKYTKNGPGAPGGQS MTSQHLKFDNSYF IYALSGAHTL GRSRPERSGHGKPETKYTKNGPGAPGGQSHTSQHLKFDNSYF IUALSGAHTL GRSRPERSGHGKPETKYTKNGPGAPGGQSHTSQHLKFDNSYP IYHLSGHHTL GRSRPERSGHGKPETKYTKNGPGAPGGQSHTSQHLKFDNSYF IYALSGAHTL GRSRPERSGHGKPETKYTKMGPGAPGGQSHTSQHLKFDNSYP IYALSGAHTL GRSRPERSGHGKPETKYTKNGPGAPGGQSHTSQHLKFDNSYF IYALSGAHTL GRSRPERSGHGKPETKYTKNGPGAPGGQSHTSQHLKFDNSYF IYALSGGHTL GRSRPERSGHGKPETKYTKNGPGAPGGQSHTSQHLKFDNSYH IYALSGAHTL GRSRPERSGHGKPETKYTKHGPGAPGGQSHTSQHLKFDNSYF $\star \star \star \star \star \star \star$

$290 \mathrm{HBD}^{300}$

MKDKFISYGLGPRQY HKDKFIAUGLGPROY MKDKFISUGLGPRQY HKDKFIAYGLGPRQL

TQEMYYLSGAHTIGG TOEMYYLSGAHTIGG TQEMYYLSGAHTIGG TQEMYYLSGAHTIGG

TQENYYLSGAHTIGG
$310 \mathrm{CBD}$

YMSAFFGPDQHAT

YMSAFFGPDOART

UMSAFFGPDQAAT

YMSAFLGPDQAFT

GFGNPNAFDHAYF

GFGNPNAFDNAY

GFGNPNAFDNAYF

GFGNPNAFDNAY

GFGNPNAFDNAYF

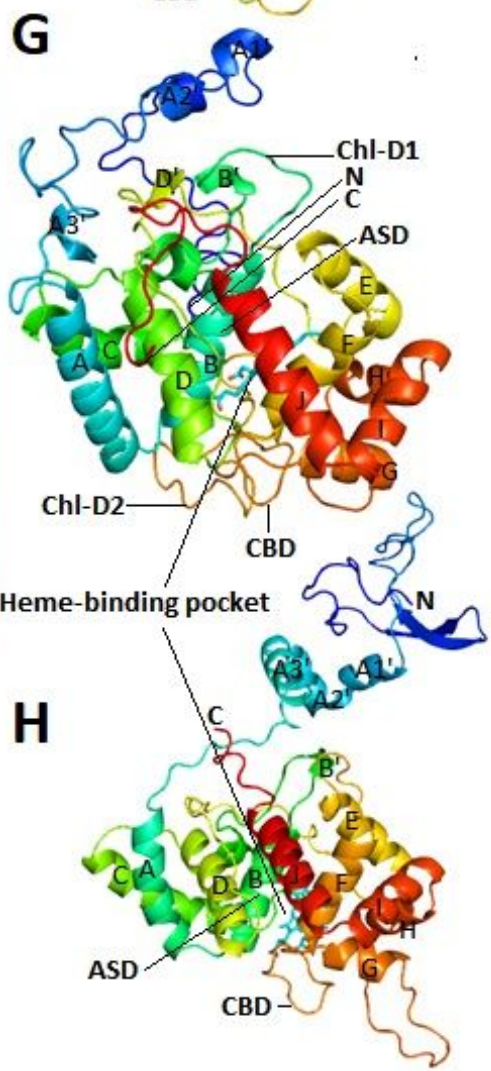

\section{Figure 5}

Protein sequence and structure analysis of TaAPXs. Multiple sequence alignments of cytosolic TaAPX protein sequences with cAPX of Pisum sativum (a), peroxisomal TaAPX proteins with AtAPX5 (b), chloroplastic TaAPX proteins with AtAPXT and AtAPXS (c), TaAPX-related (TaAPX-R) protein sequences with AtAPX4 of Arabidopsis thaliana (d). Green, blue and purple shaded boxes represents the active site domain (ASD), heme-binding domain (HBD) and cation binding domain (CBD) present in all the groups, but substituted with new sets of residues in TaAPX-R group proteins. Two additional regions chloroplastic domain 1 and 2 are highlighted with yellow shaded box and present only in chloroplastic APX proteins. Red asterisks and Green marks represent the amino-acid residues involved in heme-binding and ascorbate binding (Lys, Cys and Arg), respectively. The residues important for cytoplasmic and peroxisomal localization 
are underlined with thick green and black line, respectively. The tertiary structure of TaAPX2-B (cAPX) (e), TaAPX1-A (mAPX) (f), TaAPX3-A.1 (ChIAPX) (g) and TaAPX8-B.1 (APX-R) (h), indicating the structural differences in proteins of all the groups.
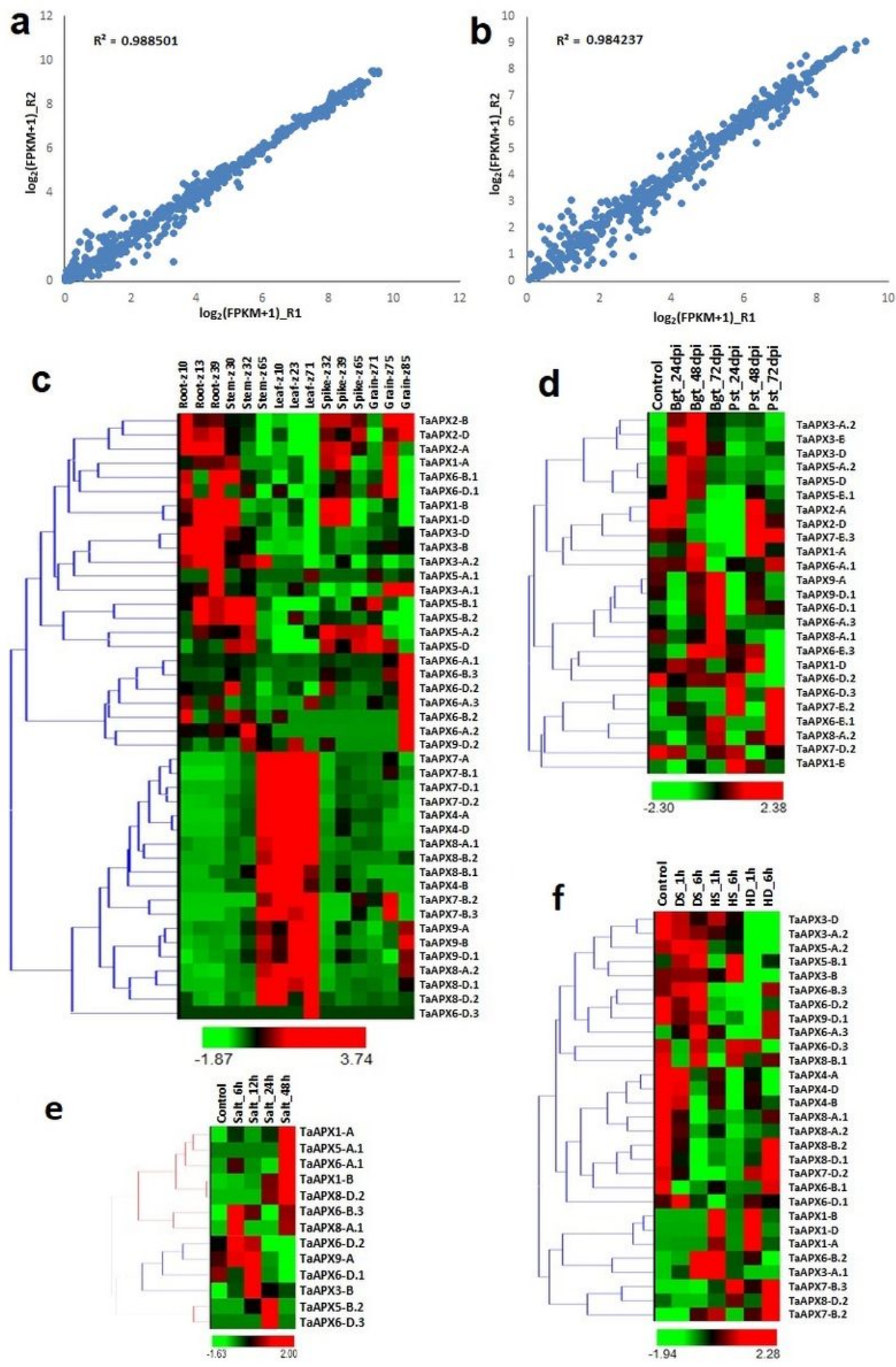

\section{Figure 6}

Correlation between the replicates of expression data in various tissue developmental stages (a) and biotic and abiotic stress conditions (b). Expression profiling of TaAPX genes in tissue developmental stages and stress conditions. Heat-map shows the expression profiles of TaAPX genes in three developmental stages 
(represented by zadoks scale) of five tissues including root, leaf, stem, spike and grain (c), biotic stress after 24, 48 and $72 \mathrm{~h}$ of Puccinia striiformis (Pst) and Blumeria graminis (Bgt) infestation (d), salt stress after 6, 12,24 and $48 \mathrm{~h}$ of treatments (e), and after 1 and $6 \mathrm{~h}$ of heat, drought and their combination stress (f). Acronyms- HS; heat stress, DS; drought stress, HD; heat and drought stress combination.
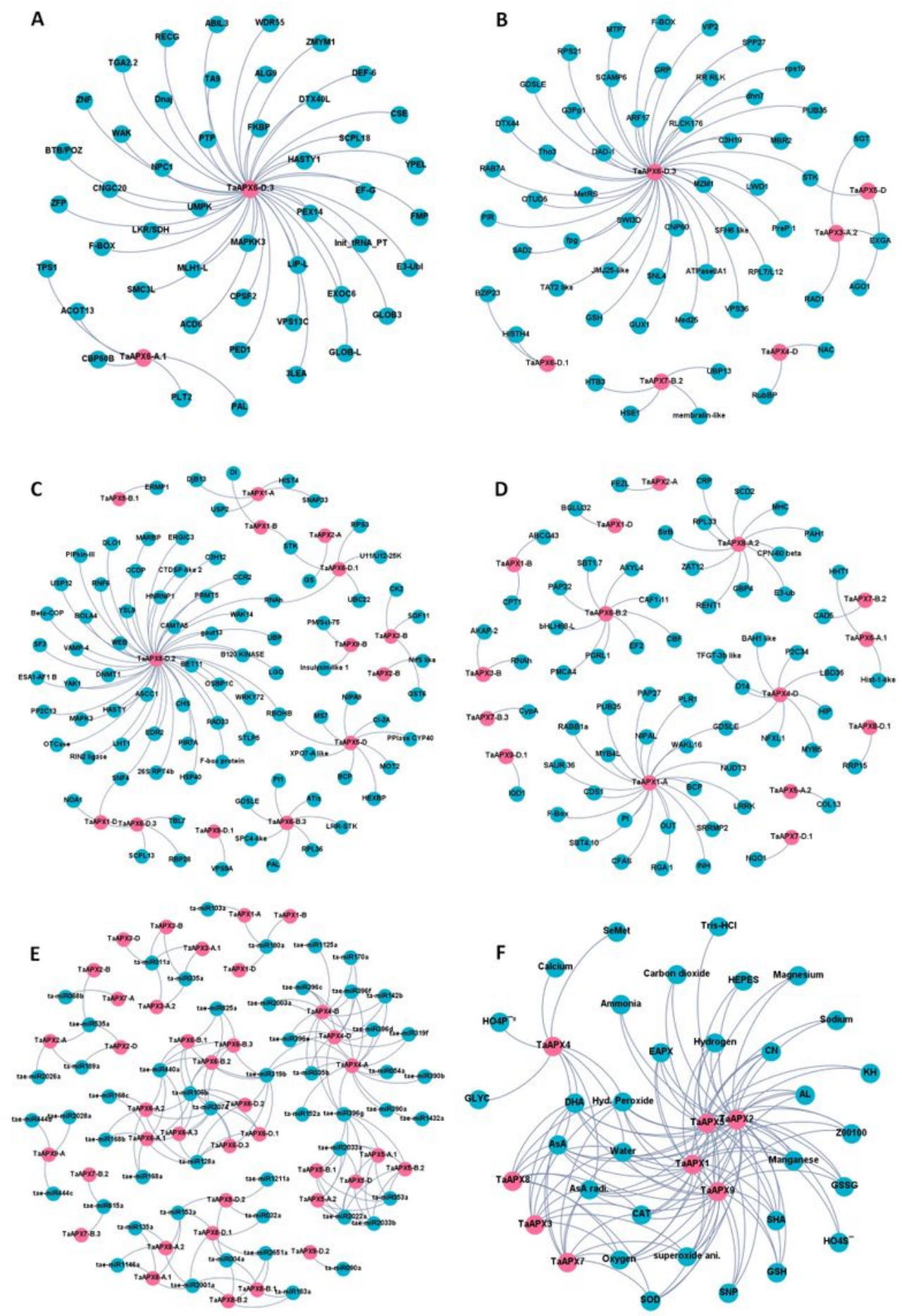

Figure 7

Co-expression analysis, miRNA targets and chemical interactions. Interaction networks of TaAPX with coexpressed genes in various tissue developmental stages (a), biotic (b), salt (c), and heat, drought and their 
combination stresses (d). Interaction of miRNA targets (e) and chemical associations (f) are also shown. The pictogram was generated using Gephi 0.9.1 software.
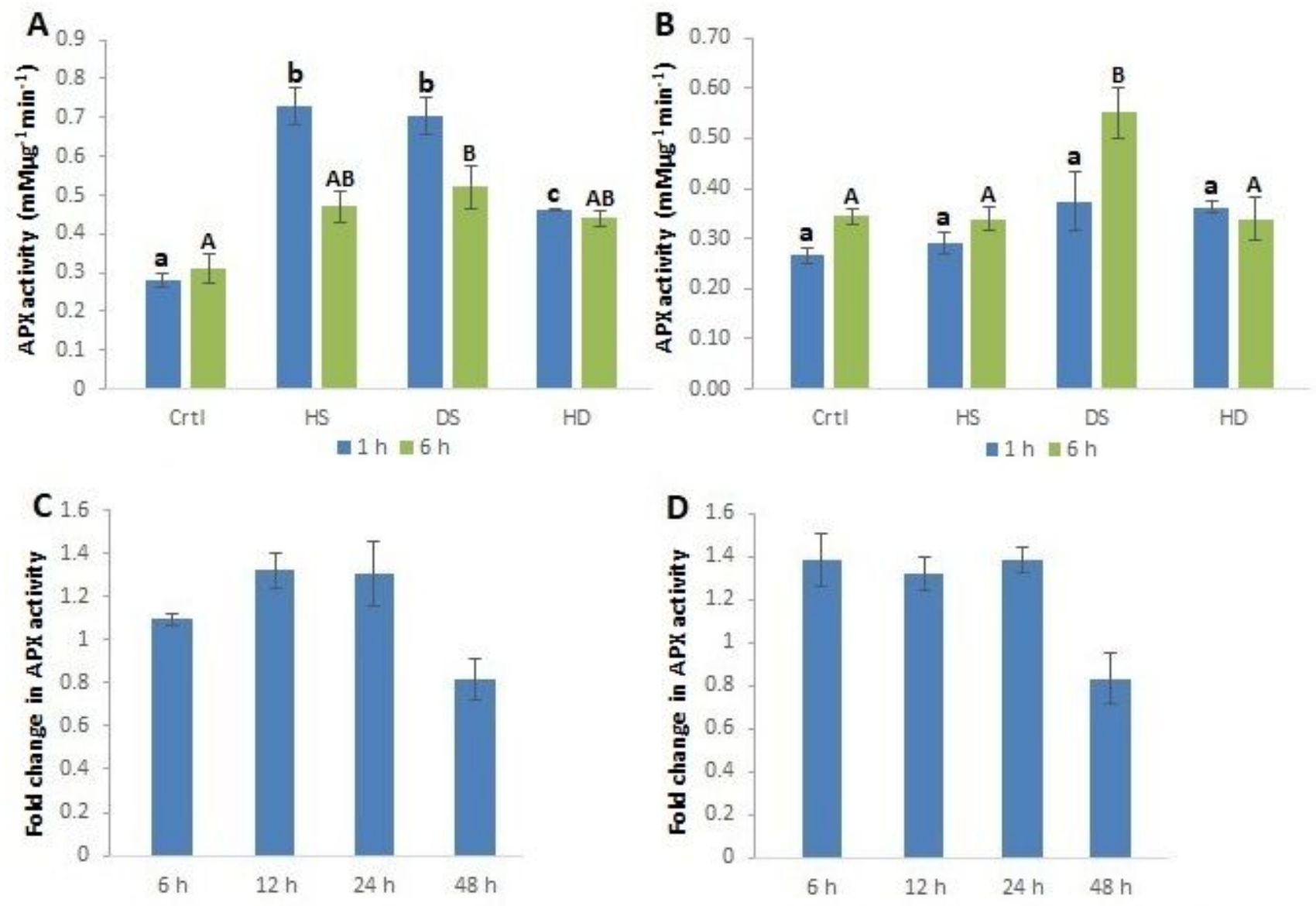

\section{Figure 8}

Ascorbate peroxidase activity during heat (HS), drought (DS) and their combination stress (HD) in shoot (a) and root (b) at $1 \mathrm{~h}$ and $6 \mathrm{~h}$ of treatments. Bars represents the mean \pm standard error of enzyme activity (mMug-1min-1). The different letters shows the significant difference $(P<0.05)$ among treatments. Fold change \pm SD in ascorbate peroxidase activity at $6,12,24$, and 48 h of salt stress in shoot (c) and root (d).

\section{Supplementary Files}

This is a list of supplementary files associated with this preprint. Click to download.

- AdditionalFileS2.xlsx

- AdditionalFileS1.xlsx

- AdditionalTable2.doc

- AdditionalFigure1.jpg

- AdditionalFileS3.xlsx

- AdditionalFigure2.jpg

- AdditionalFileS6.xIsx 
- AdditionalFileS5.xlsx

- AdditionalFileS4.xIsx

- AdditionalTable1.docx 